\title{
ON CERTAIN ELEMENTS IN THE BERNSTEIN CENTER OF GL $_{2}$
}

\author{
SANDEEP VARMA
}

\begin{abstract}
Let $F$ be a nonarchimedean local field of residue characteristic $p$, and let $r$ be an odd natural number less than $p$. Using the work of Moy and Tadić, we find an element $z$ of the Bernstein center of $G=\mathbf{G L}_{2}(F)$ that acts on any representation $\pi$ of $G$ by the scalar $z(\pi)=\operatorname{tr}\left(\right.$ Frob; $\left.\left(\operatorname{Sym}^{r} \circ \varphi_{\pi}\right)^{I_{F}}\right)$, the trace of any geometric Frobenius element Frob of the absolute Weil group $W_{F}$ of $F$, acting on the inertia-fixed points of the representation $\mathrm{Sym}^{r} \circ \varphi_{\pi}$ of $W_{F}$, where $\varphi_{\pi}: W_{F} \rightarrow \hat{G}$ is the restriction to $W_{F}$ of the Langlands parameter of $\pi$. This element $z$ is specified by giving the functions obtained by convolving it with the characteristic functions of a large class of compact open subgroups of $G$, that includes all the groups of both the congruence and the Iwahori filtrations of $G$ having depth at least one.
\end{abstract}

\section{INTRODUCTION}

Let $F$ be a nonarchimedean local field of residue characteristic $p$, and let $\mathfrak{o} \subset F$ denote its ring of integers. Let $\mathbf{G}$ denote the group $\mathbf{G L}_{2}$ over $F$, and let $G=$ $\mathbf{G L}_{2}(F)$. Let $\mathcal{Z}=\mathcal{Z}(G)$ denote the Bernstein center $([2])$ of $G$. Recall that elements of $\mathcal{Z}$ may be viewed as essentially compact invariant distributions on $G$, or equivalently, as regular functions on the Bernstein variety $\Omega(G)$ of $G$.

Let $\mathbf{r}: \hat{G} \rightarrow G L(V)$ denote a representation of the Langlands dual group $\hat{G}$ of $\mathbf{G}$. We consider the following function $z_{\mathbf{r}}$ on $\Omega(G)$. Let $\pi$ be a supercuspidal representation of $M=\mathbf{M}(F)$, for a Levi subgroup $\mathbf{M}$ of $\mathbf{G}$, and $\iota: \hat{M} \rightarrow \hat{G}$ the associated inclusion of Langlands dual groups. Let $\varphi_{\pi}: W_{F} \rightarrow \hat{M}$ denote the Langlands parameter for $\pi, W_{F}$ denoting the absolute Weil group of $F$. Since $\mathbf{G}$ is a general linear group, any subquotient of the representation $\operatorname{Ind}_{M}^{G} \pi$, Ind standing (as in the rest of this paper) for normalized induction, has a Langlands parameter whose restriction to $W_{F}$ is $\iota \circ \varphi_{\pi}$. Then to $\pi$ one attaches the scalar:

$$
z_{\mathbf{r}}(\pi):=\operatorname{tr}\left(\text { Frob }\left.\right|_{V^{I_{F}}}\right)
$$

where tr denotes trace, $I_{F} \subset W_{F}$ is the inertia subgroup, Frob denotes any geometric Frobenius element of $W_{F}$, and Frob acts on $V^{I_{F}}$ through $\mathbf{r} \circ \iota \circ \varphi_{\pi} . \pi \mapsto z_{\mathbf{r}}(\pi)$ can be easily seen to define a regular function on $\Omega(G)$, and hence gives an element $z_{\mathbf{r}} \in \mathcal{Z}=\mathcal{Z}(G)$. Since $z_{\mathbf{r}}$ is essentially compact and invariant, if $\operatorname{ch}_{K}$ denotes the measure-normalized characteristic function of a compact open subgroup $K$ of $G$, then the distribution $z_{\mathbf{r}} * \operatorname{ch}_{K}$ can be realized by an element $f_{\mathbf{r}, K}$ of $C_{c}^{\infty}(K \backslash G / K) \subset C_{c}^{\infty}(G)$.

Received by the editors December 23, 2011 and, in revised form, August 19, 2012.

2010 Mathematics Subject Classification. Primary 22E50, 22 E35.

Key words and phrases. Bernstein center, stable Bernstein center. 
Let $r$ denote a natural number. Associated to $r$ we have the $r$-th symmetric power representation $\mathbf{r}=\operatorname{Sym}^{r}: \hat{G} \rightarrow \mathbf{G L}_{r+1}(\mathbb{C})$. As per the above paragraph, attached to this $\mathbf{r}$ is an element $z_{r}=z_{\mathbf{r}} \in \mathcal{Z}$, and thence corresponding to any compact open subgroup $K$ of $G$, an element $f_{r, K} \in C_{c}^{\infty}(G)$.

When $K$ is a hyperspecial (respectively, parahoric) subgroup of $G$, the values of $f_{r, K}$ can be obtained from the Satake (respectively, Bernstein) isomorphism. For $K$ belonging to the congruence filtration of open compact subgroups of $G$, the function $f_{1, K}$ has been explicitly described by P. Scholze in Section 14 of [9]. In this note we take $r$ to be an odd natural number smaller than the residue characteristic $p$ of $F, K$ to be $K_{B}:=1+B$ for a lattice $B \subset M_{2}(\mathfrak{o})$ satisfying some reasonable assumptions (Hypothesis 2.1), and compute $f_{B}:=f_{r, K_{B}}$. For this we most crucially rely on the methods of A. Moy and M. Tadić in [6. Moy and Tadić have developed techniques to construct elements belonging to the Bernstein centers of reductive groups over nonarchimedean local fields. In [6] they give a method to construct all elements supported in any given connected component of the Bernstein variety. Their algorithm lets us explicitly construct these elements whenever we understand the characters of the representations belonging to this component well enough to integrate certain functions constructed out of them. This is the case in the situations of interest to us in this paper. Moy and Tadic also explain the sense in which elements of the Bernstein center that are supported on a single component form building blocks for more general elements. For our purposes, this comes down to convolving the distributions we get via the Moy-Tadic algorithm with $\mathrm{ch}_{K_{B}}$, and summing the resulting functions.

The results of our computation of $f_{B}$ are stated in Theorem 6.1 the main result in this paper. We do not repeat it here since the description of this function is somewhat lengthy. If val $: F \rightarrow \mathbb{Z}$ is the discrete valuation on $F$, it will turn out that $f_{B}$ is supported on the set of $g \in G$ such that val $\operatorname{det} g=r$. Some factors entering into the description of $f_{B}(g)$ are $b:=\operatorname{valtr} g, k_{B, g}:=\min \{\operatorname{val} \operatorname{tr} g x \mid x \in B\}$, and another term denoted $l_{a b}(g)$, which depends on $b$, and, roughly speaking, generalizes the function $l(g)=\operatorname{val}(1-\operatorname{tr} g+\operatorname{det} g)$ entering into the description of $f_{B}$ in Section 14 of [9] (where the case $r=1$ is considered).

For those groups $K$ in the congruence and the Iwahori filtrations of depth (as per the Moy-Prasad normalization) at least one, we recover the functions obtained by Scholze as a special case.

We briefly indicate in this paragraph why one might be interested in such functions $f_{\mathbf{r}, K}$. For this paragraph alone, we use notation from Section 11 of [4. In certain instances (i.e., for certain more general $G$, certain compact subgroups $K \subset G$ and certain representations $\mathbf{r}$ of $\hat{G}$ ), a certain constant multiple $\phi_{r}$ (the subscript $r$ here, used to make notation consistent with [4], is not the integer $r$ referred to in the rest of this note) of the function $f_{\mathbf{r}, K}$ has been observed to figure in an equation of the form:

$$
\sum_{x \in S h\left(k_{r}\right)} \operatorname{Tr}^{s s}\left(\Phi_{\mathfrak{p}}^{r}, R \Psi_{x}\left(\overline{\mathbb{Q}}_{l}\right)\right)=\sum_{\gamma_{0}} \sum_{(\gamma, \delta)} c\left(\gamma_{0} ; \gamma, \delta\right) O_{\gamma}\left(f^{p}\right) T O_{\delta \sigma}\left(\phi_{r}\right) .
$$

In particular, the left-hand side, known as the semisimple Lefschetz number, is the sum of semisimple traces of Frobenius acting on stalks of a certain nearby cycle sheaf $R \Psi\left(\overline{\mathbb{Q}}_{l}\right)$ attached to an $\mathfrak{o}$-model for a Shimura variety associated to $\mathbf{G}$. The representation $\mathbf{r}$ comes from the Shimura datum defining the Shimura variety. For exposition on this material, we refer the reader to 4], and for some related concepts 
regarding Shimura varieties with bad reduction we refer the reader to $[8$. When $K$ is a hyperspecial subgroup of $G$, this equation has been proved by Kottwitz; see [5]. When $K$ is an Iwahori subgroup of $G$, the equation has been proved by T. Haines and B.C. Ngô in [3]. For $\mathbf{G L}_{2}$ over unramified extensions of $\mathbb{Q}_{p}$, and arbitrary $K$ belonging to the congruence filtration, Scholze has proved the equation in [9], for his implementation of the Langlands-Kottwitz method for the modular curve.

We should also mention that in the context of Shimura varieties, the representations $\mathbf{r}$ that arise correspond to miniscule coweights, unlike the odd symmetric powers considered in this note (except for the standard representation). Nevertheless, this restriction does not apply to their function field analogues, so one might hope that these computations might be of some use. In addition, one may also hope that our computations might give some rough preliminary insights into the nature of these very interesting functions $f_{\mathbf{r}, K}$ in general.

The organization of this note is as follows. In Section 2 we set up some notation, and state and discuss the restrictions we impose on $B$. Then we recall that one can, as in [6], write $z_{r}=\sum z_{r, \Omega}$, the summation being over the connected components $\Omega \subset \Omega(G)$, and each $z_{r, \Omega}$ denoting the element of $\mathcal{Z}$ supported on $\Omega$ and equalling $z_{r}$ on it (here, note that although infinitely many of the $z_{r, \Omega}$ will be nonzero in general, the sum $\sum z_{r, \Omega}$ makes sense as a distribution on $G$ since for any given $\varphi \in C_{c}^{\infty}(G)$, only finitely many of the $z_{r, \Omega}(\varphi)$ will be nonzero).

Each such $z_{r, \Omega}$ can be realized by a locally integrable $G$-invariant function $f_{r, \Omega}$ on $G$. We describe in the light of this as to why $f_{B}$ is just the sum of the contributions $f_{r, \Omega} * \operatorname{ch}_{K_{B}}$ from the various components $\Omega \subset \Omega(G)$, doing in particular some preliminary set up for analyzing contributions from the principal series components. In Section 3 we prove that the supercuspidal components do not contribute to $f_{B}$. In Section 4 we analyze contributions from certain principal series components $\Omega \subset \Omega(G)$, and in Section 5 we treat the remaining components. In both cases, the $f_{r, \Omega}$ 's can be obtained by adapting the computations of [6] and [7], from the case of $\mathbf{S L}_{2}$ treated by Moy and Tadić, to the case of $\mathbf{G L}_{2}$. However, at least in Section 5, convolving them with the measure-normalized characteristic function $\operatorname{ch}_{K_{B}}$ of $K_{B}$ has needed a little bit of work. Finally, in Section 6 we put things together, computing $f_{B}$ by adding up the contributions $f_{r, \Omega} * \mathrm{ch}_{K_{B}}$ from the various components $\Omega$.

We have made use of the assumptions on $r$ (being odd and less than $p$ ) in ruling out contributions from components $\Omega$ consisting of supercuspidal representations. The assumption that $r$ is odd is crucial in Sections 4 and 5 as this ensures that for the principal series components $\Omega$ featured there, $f_{r, \Omega}$ is supported on elements of $G$ well away from the singular set. In Section 5 , this assumption also enables one to approximate a factor in $f_{r, \Omega}$ using the trace function, thereby avoiding reference to eigenvalues (see equation (5.4)), which helps us crucially in performing the convolution with $\operatorname{ch}_{K_{B}}$. That $r$ is less than $p$ is also used in Section 6 , in getting closed expressions for terms like $l_{a b}(g)$ that go into the expression for $f_{B}(g)$, for $g \in G$. It is not clear to the author if the techniques here can in any way be adapted to deal with groups of higher rank. Doing so would involve integrating functions involving supercuspidal character values near the identity and on the so called "bad shell" (even when $r=1$, one would have supercuspidal representations of Levi subgroups figuring in). Moreover, it might not be possible to adapt the aforementioned trick of expressing the $f_{r, \Omega}$ 's of Section 5 using traces, since it is 
only for $\mathbf{G L}_{2}(F)$ that the trace of an element gives so much information about its conjugacy class.

\section{Setup}

As in the introduction, let $F$ be a nonarchimedean local field of residue characteristic $p, \mathfrak{o} \subset F$ its ring of integers, $\mathfrak{p}$ the maximal ideal of $\mathfrak{o}$, and $\varpi$ a uniformizing parameter. Let val $: F \rightarrow \mathbb{Z}$ denote its usual discrete valuation, and let $|\cdot|=q^{-\operatorname{val}(\cdot) \text {, }}$ with $q=\#(\mathfrak{o} / \mathfrak{p})$, denote the usual norm on $F$. Let $W_{F}$ denote the absolute Weil group of $F$ and $I_{F} \subset W_{F}$ the inertia group.

Let $\mathbf{G}$ denote the group $\mathbf{G L}_{2}$ over $F$. Let $\mathbf{B}$ denote its Borel subgroup consisting of upper triangular matrices, and $\mathbf{A}$ its maximal torus consisting of diagonal matrices. We will write $G L_{2}, G$ and $A$ for $\mathbf{G L}_{2}(F), \mathbf{G}(F)$ and $\mathbf{A}(F)$, respectively. tr and det will stand for the trace and determinant functions on $G$, respectively. Let $\mathcal{Z}=\mathcal{Z}(G)$ denote the Bernstein center ([2]) of $G$. We follow [1] in giving $G$ the measure that gives $\mathbf{G L}_{2}(\mathfrak{o})$ volume 1, except that when we integrate over the compact set $K_{B}$ considered later below, we give $K_{B}$ its normalized Haar measure. There will not be any confusion between these choices at any point. Without further mention, we will identify $A$ with $F^{\times} \times F^{\times}$in the obvious fashion, so whenever we write $(\alpha, \beta)$ for an element of $A$, we will be referring to the diagonal matrix with $\alpha \in F^{\times}$and $\beta \in F^{\times}$in the upper left and lower right corners, respectively. Whenever we write an element of $F^{\times}$as $\varpi^{a} o$ it will be implicitly assumed that $a \in \mathbb{Z}$ and that $o \in \mathfrak{o}^{\times}$.

Let $r$ be an odd natural number with $r<p$. For a supercuspidal representation $\pi$ of $M:=\mathbf{M}(F)$, where $\mathbf{M}(=\mathbf{A}$ or $\mathbf{G})$ is a Levi subgroup of $\mathbf{G}$, we define $z_{r}(\pi)$ to be the trace of any Frobenius element of $W_{F}$ acting on $V^{I_{F}}$, where $V$ is the representation space of $\operatorname{Sym}^{r} \circ \varphi_{\pi}, \varphi_{\pi}$ being a Langlands parameter for $\pi$ viewed as a map $W_{F} \rightarrow \hat{G}$ (after composition with the inclusion $\hat{M} \rightarrow \hat{G}$ ). From general results about the Bernstein center, it easily follows that there exists an element $z_{r} \in \mathcal{Z}$ such that for any supercuspidal representation $\pi$ of $M:=\mathbf{M}(F)$ for a Levi subgroup $\mathbf{M}$ of $\mathbf{G}, z_{r}$ acts on all subquotients of $\operatorname{Ind}_{M}^{G} \pi$ by the scalar $z_{r}(\pi)$.

Let $B$ be a lattice in $\mathbf{M}_{2}(F)$ such that $K_{B}:=1+B$ is a subgroup of $\mathbf{G L}_{2}(F)$. We will be considering the measure on $K_{B}$ giving it volume 1 . For any $g \in G$, let $k_{B, g}$ and $m_{B}$ be the integers such that the fractional ideals

$$
\{\operatorname{tr} g x \mid x \in B\} \text { and }\{\operatorname{tr} x \mid x \in B\}
$$

in $F$ equal $\mathfrak{p}^{k_{B, g}}$ and $\mathfrak{p}^{m_{B}}$, respectively.

We make the following assumptions on $B$.

Hypothesis 2.1. We assume that $K_{B}:=1+B$ is a subgroup of $\mathbf{G L}_{2}(F)$, and that:

(i) For all $g \in G$ conjugate to some $\left(\varpi^{a} o_{1}, \varpi^{b} o_{2}\right)$,

$$
\{\operatorname{tr} g x \mid x \in B\} \supset\{\operatorname{tr} g \cdot \operatorname{tr} x \mid x \in B\} .
$$

(ii) $B$ is small enough so that $m_{B}>0$, and so that for all $x \in B$, $\operatorname{det} x \in$ $\varpi^{m_{B}+1} \mathfrak{o}$.

(iii) $\operatorname{det}\left(I_{2}+\varpi^{m_{B}} B^{\perp}\right) \subset \mathfrak{o}^{\times}$, where $I_{2}$ denotes the two by two identity matrix and

$$
B^{\perp}=\left\{y \in \mathbf{M}_{2}(F) \mid \operatorname{tr} x y \in \mathfrak{p}\right\} .
$$


Remark 2.2. Suppose $B$ is of the form $B_{n}:=\varpi^{n} \mathbf{M}_{2}(\mathfrak{o})$ for some $n \in \mathbb{N}$, or of the form:

$$
B_{\mathcal{L}, n}:=\left\{x \in \mathbf{M}_{2}(\mathfrak{o}) \mid x L_{j} \subset L_{j+n} \quad \forall j \in \mathbb{Z}\right\},
$$

for some $n \in \mathbb{N} \backslash\{1\}$ and lattice chain $\mathcal{L}=\left\{L_{j}\right\}_{j \in \mathbb{Z}}$. We claim that $B$ satisfies (i)-(iii). First note that any lattice chain $\mathcal{L}$ may be $G$-conjugated to the standard lattice chain $\mathcal{L}_{0}$. Therefore, to prove (i)-(iii) for $B$ of the form $B_{\mathcal{L}, n}$ as above, it suffices to treat the case where $\mathcal{L}=\mathcal{L}_{0}$. Moreover, we have an explicit description for $B_{\mathcal{L}_{0}, n}$ :

$$
B_{\mathcal{L}_{0}, n}=\left(\begin{array}{cc}
\mathfrak{p}^{\lceil n / 2\rceil} & \mathfrak{p}^{\lceil(n-1) / 2\rceil} \\
\mathfrak{p}^{\lceil(n+1) / 2\rceil} & \mathfrak{p}^{\lceil n / 2\rceil}
\end{array}\right)
$$

Thus, we may and do assume that $B=B_{n}$ for some $n \in \mathbb{N}$ or $B_{\mathcal{L}_{0}, n}$ for some $n \in \mathbb{N} \backslash\{1\}$. Then (i) follows from the observation that whenver $x \in B$, the element $(\operatorname{tr} x, \operatorname{tr} x) \in A$ also lies in $B$. (ii) is straightforward given the explicit description of $B_{\mathcal{L}_{0}, n}$, and (iii) follows from $\varpi^{m_{B_{n}}} B_{n}^{\perp}=\varpi^{n} B_{1-n}=B_{1}$ and

$$
\varpi^{m_{B} \mathcal{L}_{0}, n} B_{\mathcal{L}_{0}, n}^{\perp}=\varpi^{\lceil n / 2\rceil} B_{\mathcal{L}_{0}, 1-n}=B_{\mathcal{L}_{0}, 1+2\lceil n / 2\rceil-n} \subset B_{\mathcal{L}_{0}, 1} .
$$

Another way to see this would be to note that lattices of the form $B_{n}(n \in \mathbb{N})$ as well as those of the form $B_{\mathcal{L}, n}(n \in \mathbb{N}, n>1)$ are Moy-Prasad lattices of the form $\mathfrak{g}_{x, r}$ for some $x$ in the Bruhat-Tits building of $G$ and some $r \geq 1$. One can still prove (i) the same way as above, while (ii) would follow from, say, choosing coordinates for an appropriate apartment and making an easy computation by hand, and (iii) would follow from the dual of $\mathfrak{g}_{x, r}$ being $\mathfrak{g}_{x,(-r)+}^{*}$ together with the fact that the trace form identifies each $\mathfrak{g}_{x, s}$ with $\mathfrak{g}_{x, s}^{*}$. Our restriction $r \geq 1$ cannot be replaced by $r>0$ without hurting (ii), and it is not clear to the author if (ii) might be modified without affecting Lemma 5.1 below in a certain, however, very special case.

Let $\operatorname{ch}_{K_{B}}$ denote the measure-normalized characteristic function of $K_{B}=1+B$. Our aim in this note will be to compute the function $f_{B}$ on $G$ that realizes the distribution $z_{r} * \operatorname{ch}_{K_{B}}$.

Let $\Omega(G)$ denote the Bernstein variety of $G$. We will often refer to connected components $\Omega \subset \Omega(G)$ as Bernstein components. Let $\Omega \subset \Omega(G)$ be such a component. Consider the element $z_{\Omega} \in \mathcal{Z}$, supported on $\Omega$ and equalling $z_{r}$ on $\Omega$. It was denoted $z_{r, \Omega}$ in Section 1 , but we are suppressing the $r$ to lighten notation. According to $\left[\underline{6}\right.$, we have that $z_{\Omega}$ is given by a locally integrable function $f_{\Omega}$ on $G$, and that $z_{r}$ is the sum of all the $z_{\Omega}$, in the sense that for all $\varphi \in C_{c}^{\infty}(G)$,

$$
\sum_{\Omega} z_{\Omega}(\varphi)=z_{r}(\varphi)
$$

with the left-hand side actually a finite sum. Note that for all $\Omega$, the distribution $z_{\Omega} * \operatorname{ch}_{K_{B}}$ is given by the function $f_{B, \Omega}$, where for all $g \in G$,

$$
f_{B, \Omega}(g)=\int_{K_{B}} f_{\Omega}(g k) d k=\int_{B} f_{\Omega}(g(1+x)) d x,
$$

where $d k$ and $d x$ denote the normalized Haar measures on $K_{B}$ and $B$, respectively.

Notation 2.3. Henceforth, we will use the shorthand notation $g_{x}:=g(1+x)$ for all $g \in G$ and $x \in B$. 
Notation 2.4. For any character $\lambda$ of $\mathfrak{o}^{\times}$, we denote by $f(\lambda)$ the conductor of $\lambda$, so $f(\lambda)$ is the smallest $n \in \mathbb{N} \cup\{0\}$ such that $\lambda$ is trivial on $U_{n}$, where $U_{n}$ equals $1+\mathfrak{p}^{n}$ if $n \in \mathbb{N}$ and $\mathfrak{o}^{\times}$if $n=0$.

Now we describe the plan of our computation. Let $f_{\mathrm{sc}, B}$ denote the sum of all $f_{B, \Omega}$ as $\Omega$ varies over the components of $\Omega(G)$ that correspond to supercuspidal representations of $G$. We will show in the next section that:

$$
f_{\mathrm{sc}, B}=0,
$$

by showing that $f_{\Omega}=0$ for all components $\Omega$ consisting of supercuspidal representations of $G$.

To proceed further, let us recall how Bernstein components consisting of principal series representations of $G$ are indexed by unordered pairs of characters of $\mathfrak{o}^{\times}$. For convenience, we will often implicitly treat characters of $\mathfrak{o}^{\times}$also as characters on the whole of $k^{\times}$, by declaring them to be trivial on $\varpi$. Given characters $\mu, \nu$ of $\mathfrak{o}^{\times}$and $s_{1}, s_{2} \in \mathbb{C}^{\times}$we define $\pi_{\mu, \nu, s_{1}, s_{2}}$ to denote the representation of $G$, unitarily induced from the character of $B$ that equals the character:

$$
\left(\mu s_{1}^{\operatorname{val}(\cdot)}, \nu s_{2}^{\operatorname{val}(\cdot)}\right):\left(\varpi^{a} o_{1}, \varpi^{b} o_{2}\right) \mapsto \mu\left(o_{1}\right) s_{1}^{a} \cdot \nu\left(o_{2}\right) s_{2}^{b}
$$

on $A$. Then the Bernstein component corresponding to an unordered pair/multiset consisting of characters $\{\mu, \nu\}$ of $\mathfrak{o}^{\times}$may be identified with $\mathbb{C}^{\times} \times \mathbb{C}^{\times}$in case $\mu \neq \nu$, and with $\left(\mathbb{C}^{\times} \times \mathbb{C}^{\times}\right) / \sim, \sim$ being the equivalence relation $\left(s_{1}, s_{2}\right) \sim\left(s_{2}, s_{1}\right)$, if $\mu=\nu$. In either case, $\left(s_{1}, s_{2}\right)$ corresponds to the (the conjugacy class of the trivially supercuspidal) representation $\left(\mu s_{1}^{\operatorname{val}(\cdot)}, \nu s_{2}^{\operatorname{val}(\cdot)}\right)$ of $A$.

Since $\mathbf{A}$ has been identified with $\mathbb{G}_{m} \times \mathbb{G}_{m}$, the Langlands dual of $\mathbf{A}$ can and will naturally be identified with $\mathbb{C}^{\times} \times \mathbb{C}^{\times}$. Thus, the Langlands parameter $\varphi_{\mu, \nu, s_{1}, s_{2}}$ corresponding to this representation $\left(\mu s_{1}^{\operatorname{val}(\cdot)}, \nu s_{2}^{\operatorname{val}(\cdot)}\right)$ of $A$ is obtained by composing the natural homomorphism $W_{F} \rightarrow F^{\times} \times F^{\times}$(obtained from the Abelianization homomorphism $W_{F} \rightarrow F^{\times}$that sends a geometric Frobenius to a uniformizing parameter in $\mathfrak{o})$ with $\left(\mu s_{1}^{\operatorname{val}(\cdot)}, \nu s_{2}^{\operatorname{val}(\cdot)}\right)$. Then as representations of $W_{F}$,

$$
\operatorname{Sym}^{r} \circ \varphi=\bigoplus_{a+b=r} \varphi_{\mu^{a}, \nu^{b}, s_{1}^{a}, s_{2}^{b}}
$$

Hence,

$$
\left(\operatorname{Sym}^{r} \circ \varphi\right)^{I_{F}}=\bigoplus_{\substack{a+b=r \\ \mu^{a} \nu^{b}=1}} \varphi_{\mu^{a}, \nu^{b}, s_{1}^{a}, s_{2}^{b}} .
$$

Therefore, for any geometric Frobenius element Frob $\in W_{F}$, and any $s_{1}, s_{2} \in \mathbb{C}^{\times}$:

$$
\operatorname{tr}\left(\operatorname{Frob} ;\left(\operatorname{Sym}^{r} \circ \varphi\right)^{I_{F}}\right)=\sum_{\substack{a+b=r \\ \mu^{a} \nu^{b}=1}}\left(\mu^{a} s_{1}^{a \operatorname{val}(\cdot)} \cdot \nu^{b} s_{2}^{b \operatorname{val}(\cdot)}\right)(\varpi)=\sum_{\substack{a+b=r \\ \mu^{a} \nu^{b}=1}} s_{1}^{a} s_{2}^{b} .
$$

We will need to treat the cases $\mu=\nu$ and $\mu \neq \nu$ separately. If $\mu=\nu=\lambda$, say, we let $z_{\lambda}$ be the element of $\mathcal{Z}$ supported in the Bernstein component of the multiset $\{\lambda, \lambda\}$, and acting on the subquotients of $\pi_{\lambda, \lambda, s_{1}, s_{2}}$ by $s_{1}^{r}+s_{1}^{r-1} s_{2}+\cdots+s_{2}^{r}$. Let $f_{\lambda, \lambda}$ be the locally integrable function as in [6] realizing the distribution $z_{\lambda}$. For $\mu \neq \nu$, given $a, b \in \mathbb{N} \cup\{0\}$ with $a+b=r$, we let $z_{\mu, \nu, a, b}$ be the element of $\mathcal{Z}$ supported on the Bernstein component of the multiset $\{\mu, \nu\}$, that acts on $\pi_{\mu, \nu, s_{1}, s_{2}}$ by $s_{1}^{a} s_{2}^{b}$. 
Let $f_{\mu, \nu, a, b}$ be the locally integrable function as in [6] that realizes the distribution $z_{\mu, \nu, a, b}$.

From Lemma 5.1 below it will follow that $f_{B, \Omega}=0$ for almost all components $\Omega \subset \Omega(G)$, so that $f_{B}$ is simply the sum of all $f_{B, \Omega}$ 's. Then from equations (2.2) and (2.3) it will follow that:

$$
f_{B}=\sum_{\lambda^{r}=1} f_{\lambda, \lambda} * \operatorname{ch}_{K_{B}}+\sum_{\substack{\mu \neq \nu \\ \underbrace{a+b=r} \\ \mu^{a} \nu^{b}=1}} f_{\mu, \nu, a, b} * \operatorname{ch}_{K_{B}} .
$$

In other words,

$$
f_{B}=f_{B}^{(0)}+f_{B}^{(>0)}
$$

where

$$
f_{B}^{(0)}=\sum_{\lambda^{r}=1} f_{\lambda, \lambda} * \operatorname{ch}_{K_{B}}
$$

and

$$
f_{B}^{(>0)}=\sum_{a+b=r} \sum_{\substack{\mu \neq \nu \\ \mu^{a} \nu^{b}=1}} f_{\mu, \nu, a, b} * \operatorname{ch}_{K_{B}}=\sum_{a+b=r} f_{B}^{(>0, b)}
$$

where

$$
f_{B}^{(>0, b)}=\sum_{\substack{\mu \neq \nu \\ \mu^{a} \nu^{b}=1}} f_{\mu, \nu, a, b} * \operatorname{ch}_{K_{B}}
$$

(whenever we refer to $f_{B}^{(>0, b)}$, it will be understood that $b \in \mathbb{Z} \cap[0, r]$ and that $a=r-b)$.

\section{Proving that supercuspidals do not Contribute}

3.1. Ruling out the exceptional supercuspidals. Even without our assumptions on $p$, the assumption that $r$ is odd enables us to prove that the exceptional supercuspidal representations do not contribute to $f_{B}$. In this subsection we give this proof. Let $\pi$ be an exceptional supercuspidal representation of $G$, and $\varphi_{\pi}: W_{F} \rightarrow \mathbf{G L}_{2}(\mathbb{C})$ an associated Langlands parameter. We wish to prove that the restriction of $\operatorname{Sym}^{r} \circ \varphi_{\pi}$ to the inertia group $I_{F} \subset W_{F}$ does not contain the trivial representation. Since $\pi$ is exceptional, the image of $\varphi_{\pi}\left(W_{F}\right)$ in $\mathbf{P G L}_{2}(\mathbb{C})$ contains $A_{4}$, so that the image in $\mathbf{P G L} \mathbf{G}_{2}(\mathbb{C})$ of $\varphi_{\pi}\left(\left[W_{F}, W_{F}\right]\right) \subset \varphi_{\pi}\left(I_{F}\right)$ contains a copy of $(\mathbb{Z} / 2 \mathbb{Z}) \times(\mathbb{Z} / 2 \mathbb{Z})$. Let $x \in \varphi_{\pi}\left(\left[W_{F}, W_{F}\right]\right) \subset \mathbf{S L}_{2}(\mathbb{C})$ be such that the image of $x$ in $\mathbf{P G L}_{2}(\mathbb{C})$ is nontrivial. Then $x^{2}$ is a central element in $\mathbf{G L}_{2}(\mathbb{C})$ while $x$ is not central, so the eigenvalues of $x$ are of the form $b,-b$ with $b \in \mathbb{C}$. But since $x$ has determinant $1, b= \pm i$. But then the eigenvalues of $\operatorname{Sym}^{r} x$ are all $\pm i$, and hence $\operatorname{Sym}^{r} x \in \operatorname{Sym}^{r} \circ \varphi_{\pi}\left(I_{F}\right)$ cannot fix any nonzero vector in the space of $\mathrm{Sym}^{r} \circ \varphi_{\pi}$.

3.2. Weil representations. Let $E / F$ be a quadratic extension and let $\pi$ be a supercuspidal representation of $G$ with Langlands parameter $\varphi_{\pi}:=\operatorname{Ind}_{W_{E}}^{W_{F}} \lambda$, where $\lambda$ is viewed as a character of $W_{E}$ via the abelianization map $W_{E} \rightarrow E^{\times}$.

Let $V_{\mathrm{Sym}^{r} \circ \varphi_{\pi}}$ denote the representation space of $\operatorname{Sym}^{r} \circ \varphi_{\pi}$, and let $I_{E} \subset I_{F}$ be the inertia group of $E$. We will show that the trace of any Frobenius element of $W_{F}$ acting on $V_{\mathrm{Sym}^{r} \circ \varphi_{\pi}}^{I_{F}}$ is 0 , so that $\pi$ and hence its Bernstein component 
does not contribute to $f_{B}$. By Frobenius reciprocity, since $\mathrm{Sym}^{r} \circ \varphi_{\pi}$ is completely irreducible, we have a decomposition (we are using here that $r$ is odd),

$$
\operatorname{Sym}^{r} \circ \varphi_{\pi}=\bigoplus_{\substack{a+b=r \\ b<a}} \operatorname{Ind}_{W_{E}}^{W_{F}} \lambda^{a}\left(\lambda^{\sigma}\right)^{b} .
$$

Let us denote the representation space of $\operatorname{Ind}_{W_{E}}^{W_{F}} \lambda^{a}\left(\lambda^{\sigma}\right)^{b}$ by $V_{b}$. Then:

$$
V_{\mathrm{Sym}^{r} \circ \varphi_{\pi}}^{I_{E}}=\bigoplus_{\substack{b=\left.0 \\ \lambda^{a}\left(\lambda^{\sigma}\right)^{b}\right|_{I_{E}}=1}}^{\lfloor r / 2\rfloor} V_{b}
$$

(where we have written $a$ for $r-b$ ).

First consider the case where $E / F$ is unramified. Here $I_{F}=I_{E}$. By equation (3.1), $V_{\mathrm{Sym}^{r} \circ \varphi_{\pi}}^{I_{E}}$, as a representation of $W_{F}$, is a direct sum of representations induced from $W_{E}$, and hence has its character supported on the $W_{F}$ conjugates of $W_{E}$ in $W_{F}$, namely, on $W_{E}$. Thus any Frobenius element, which necessarily lies outside $W_{E}$, acts on $V_{\mathrm{Sym}^{r} \circ \varphi_{\pi}}^{I_{E}}=V_{\mathrm{Sym}^{r} \circ \varphi_{\pi}}^{I_{F}}$ by a trace zero map.

Note. Another way to see this is to make computations following [6], upon which it will follow that the contribution of the Bernstein component consisting of the representations $\left\{\pi \otimes s^{\text {val } \circ \operatorname{det}(\cdot)} \mid s \in \mathbb{C}^{\times}\right\}$, for an unramified unitary supercuspidal representation $\pi$ of $G$, is a constant multiple of $\check{\Theta}_{\pi}$ restricted to $\{g \in G \mid \operatorname{val}(\operatorname{det} g)=r\}$, where $\check{\Theta}_{\pi}(g)=\Theta_{\pi}\left(g^{-1}\right) \forall g \in G, \Theta_{\pi}$ denoting the character of $\pi$. But unramified supercuspidal representations are induced from $\mathbf{G L}_{2}(\mathfrak{o}) \cdot \mathbf{Z}_{\mathbf{G}}(F)$, and hence have characters supported on the set of elements $g$ with val(det $g)$ even.

Now consider the case where $E / F$ is ramified. Let $0 \leq b \leq\lfloor r / 2\rfloor$. It is enough to show that $\lambda^{a}\left(\lambda^{\sigma}\right)^{b}$ cannot be trivial on $I_{E}$. Suppose it were. Then $\lambda^{a}\left(\lambda^{\sigma}\right)^{b}$ is trivial on $\mathfrak{o}_{E}^{\times}$, the group of units in the ring $\mathfrak{o}_{E}$ of integers in $E$. Therefore if $E^{1}$ denotes the kernel of the norm map from $E^{\times}$to $F^{\times}, \lambda^{a-b}$ is trivial on $E^{1}$. However, since $E / F$ is ramified, the order of any nontrivial continuous character of $E^{1}$ cannot have any factor other than 2 and $p$. Since $a-b$ is both odd and relatively prime to $p$ (as $r<p$ ), it follows that $\lambda$ is trivial on $E^{1}$, which cannot be the case as $\pi$ is supercuspidal.

Thus, we have proved equation (2.2).

\section{UNRAMIFIED PRINCIPAL SERIES COMPONENTS}

Let $\lambda$ be any character of $\mathfrak{o}^{\times}$with $\lambda^{r}=1$. The Bernstein component of $(\lambda, \lambda)$ can then be identified with $\mathbb{C}^{\times} \times \mathbb{C}^{\times} / \sim$ where $\left(s_{1}, s_{2}\right) \sim\left(s_{2}, s_{1}\right)$.

Let $\mathrm{St}_{\lambda}$ denote the usual Steinberg representation tensored with $\lambda \circ$ det. In other words, $\mathrm{St}_{\lambda}$ is the quotient of $\operatorname{Ind}_{B}^{G}\left(\lambda|\cdot|^{-1 / 2}, \lambda|\cdot|^{1 / 2}\right)=\pi_{\lambda, \lambda, \sqrt{q}, \sqrt{q}^{-1}}$ by its unique subrepresentation, namely $\lambda \circ$ det. The cuspidal support of $\mathrm{St}_{\lambda}$ is the $G$-orbit of $\left(A,\left(\lambda|\cdot|^{1 / 2}, \lambda|\cdot|^{-1 / 2}\right)\right)$. For $s \in \mathbb{C}^{\times}$, let $\mathrm{St}_{\lambda, s}$ denote $\mathrm{St}_{\lambda} \otimes s^{\operatorname{val} o \operatorname{det}(\cdot)}$. The $\mathrm{St}_{\lambda, s}$ are the only discrete series representations of $G L_{2}$ having cuspidal support in the Bernstein component corresponding to $(\lambda, \lambda)$.

Recall that we have let $z_{\lambda}$ be the element of $\mathcal{Z}(G)$ that is supported on the Bernstein component of $(\lambda, \lambda)$, and which for $s_{1}, s_{2} \in \mathbb{C}^{\times}$acts on $\pi_{\lambda, \lambda, s_{1}, s_{2}}$ by the scalar $z_{\lambda}\left(\pi_{\lambda, \lambda, s_{1}, s_{2}}\right):=s_{1}^{r}+s_{1}^{r-1} s_{2}+\cdots+s_{2}^{r}$. Since $\mathrm{St}_{\lambda, s}$ is a subquotient of 
$\pi_{\lambda, \lambda, \sqrt{q} s, \sqrt{q}^{-1} s}$, it follows that $z_{\lambda}$ acts on $\mathrm{St}_{\lambda, s}$ by the constant

$$
z_{\lambda}\left(\mathrm{St}_{\lambda, s}\right)=(\sqrt{q} s)^{r}+(\sqrt{q} s)^{r-1}\left(\sqrt{q^{-1}} s\right)+\cdots+\left(\sqrt{q^{-1}} s\right)^{r}=\frac{q^{r+1}-1}{(q-1) q^{r / 2}} \cdot s^{r} .
$$

Now we apply the usual Plancherel inversion formula in Remark 2.17 of 2 . We follow the more explicit form given in equation (2.4.3) of $[6$, except that we use notation from Theorem 2.1 of [1]. Thus, we get that for all $\varphi \in C_{c}^{\infty}(G)$ :

$$
\begin{aligned}
z_{\lambda}(\varphi)= & c(G \mid A)^{-2} \gamma(G \mid A)^{-1} \frac{1}{2}\left(\frac{1}{2 \pi i}\right)^{2} \int_{\left|s_{1}\right|=\left|s_{2}\right|=1} z_{\lambda}\left(\pi_{\lambda, \lambda, s_{1}, s_{2}}\right) \\
& \cdot \mu_{G \mid A}\left(\lambda s_{1}^{\operatorname{val}(\cdot)}, \lambda s_{2}^{\operatorname{val}(\cdot)}\right) d\left(\lambda s_{1}^{\operatorname{val}(\cdot)}, \lambda s_{2}^{\operatorname{val}(\cdot)}\right) \\
& \cdot \check{\Theta}_{\lambda, \lambda, s_{1}, s_{2}}(\varphi) \frac{d s_{1}}{s_{1}} \frac{d s_{2}}{s_{2}}+c(G \mid G)^{-2} \gamma(G \mid G)^{-1} \frac{1}{1} \\
& \cdot \frac{1}{2 \pi i} \int_{|s|=1} z_{\lambda}\left(\operatorname{St}_{\lambda, s}\right) \mu_{G \mid G}\left(\operatorname{St}_{\lambda, s}\right) d\left(\operatorname{St}_{\lambda, s}\right) \cdot \check{\Theta}_{S t, \lambda, s}(\varphi) \cdot 2 \frac{d s}{s},
\end{aligned}
$$

where $\check{\Theta}_{\lambda, \lambda, s_{1}, s_{2}}$ stands for the character of $\pi_{\lambda, \lambda, s_{1}, s_{2}}$ composed with $g \mapsto g^{-1}$, and $\check{\Theta}_{S t, \lambda, s}$ stands for the character of $\mathrm{St}_{\lambda, s}$ composed with $g \mapsto g^{-1}$. Here, the choice of measures is justified by the discussion in the latter half of Page 31 of [1]. The 2 in $2 d s / s$ comes from the normalization of measures in [11] (see the last sentence of page 239 there), which [1] follows. We are also using that the various $\mathrm{St}_{\lambda, s}$ are all pairwise distinct, and this well-known fact may be seen either from Langlands parametrization or the character of $\mathrm{St}_{\lambda, s}$, recalled later.

$\mu_{G \mid G}, c_{G \mid G}$ and $\gamma_{G \mid G}$ are trivial. The formal degree of the usual Steinberg representation, and hence of each $\mathrm{St}_{\lambda, s}$, with respect to the measure we have chosen is $(q-1) / 2$ (see, e.g., page 28 of [1]). Moreover, $\gamma(G \mid A)=(q+1) / q$ and $c(G \mid A)=1$ (e.g., equations (3) and (4) of [1]). From Theorem 4.5 of [1], we have:

$$
\mu_{G \mid A}\left(\lambda s_{1}^{\operatorname{val}(\cdot)}, \lambda s_{2}^{\operatorname{val}(\cdot)}\right)=(q+1)^{2} \frac{\left(s_{1}-s_{2}\right)^{2}}{\left(q s_{1}-s_{2}\right)\left(s_{1}-q s_{2}\right)} .
$$

Plugging the formulas summarized into the above paragraph and the formulas for $z_{\lambda}\left(\pi_{\lambda, \lambda, s_{1}, s_{2}}\right)$ and $z_{\lambda}\left(\mathrm{St}_{\lambda, s}\right)$ into equation (4.1), we get:

$$
\begin{aligned}
z_{\lambda}(\varphi)= & \frac{q}{(q+1)} \cdot \frac{1}{2} \cdot\left(\frac{1}{2 \pi i}\right)^{2} \int_{\left|s_{1}\right|=\left|s_{2}\right|=1}\left(s_{1}^{r}+s_{1}^{r-1} s_{2}+\cdots+s_{2}^{r}\right) \\
& \cdot(q+1)^{2} \frac{\left(s_{1}-s_{2}\right)^{2}}{\left(q s_{1}-s_{2}\right)\left(s_{1}-q s_{2}\right)} \cdot\left(\int_{G} \varphi(g) \check{\Theta}_{\lambda, \lambda, s_{1}, s_{2}}(g) d g\right) \frac{d s_{1}}{s_{1}} \frac{d s_{2}}{s_{2}} \\
& \quad+\frac{1}{2 \pi i} \int_{|s|=1}\left(\frac{q^{r+1}-1}{(q-1) q^{r / 2}} \cdot s^{r}\right) \cdot \frac{q-1}{2} \cdot\left(\int_{G} \varphi(g) \check{\Theta}_{S t, \lambda, s}(g) d g\right) 2 \frac{d s}{s} \\
= & \int_{G} \varphi(g)\left(\frac{q(q+1)}{2}\left(\frac{1}{2 \pi i}\right)^{2} \int_{\left|s_{1}\right|=\left|s_{2}\right|=1} \cdot \frac{\left(s_{1}^{r}+\cdots+s_{2}^{r}\right) \check{\Theta}_{\lambda, \lambda, s_{1}, s_{2}}(g)}{\left(q s_{1}-s_{2}\right)\left(s_{1}-q s_{2}\right)} \frac{d s_{1}}{s_{1}} \frac{d s_{2}}{s_{2}}\right) d g \\
& +\int_{G} \varphi(g)\left(\frac{q^{r+1}-1}{q^{r / 2}} \frac{1}{2 \pi i} \int_{|s|=1} s^{r} \check{\Theta}_{S t, \lambda, s}(g) \frac{d s}{s}\right) d g,
\end{aligned}
$$


by Fubini's theorem. We thus have a locally integrable function $f_{\lambda, \lambda}=f_{\lambda, \lambda}^{\prime}+f_{\lambda, \lambda}^{\prime \prime}$ that realizes the distribution $z_{\lambda}$ on $G$, where for all $g \in G, f_{\lambda, \lambda}^{\prime}(g)$ equals:

$$
\begin{aligned}
f_{\lambda, \lambda}^{\prime}(g)= & \frac{q(q+1)}{2(2 \pi i)^{2}} \int_{\left|s_{1}\right|=\left|s_{2}\right|=1}\left(s_{1}^{r}+\cdots+s_{2}^{r}\right) \check{\Theta}_{\lambda, \lambda, s_{1}, s_{2}}(g) \\
& \cdot \frac{\left(s_{1}-s_{2}\right)^{2}}{\left(q s_{1}-s_{2}\right)\left(s_{1}-q s_{2}\right)} \frac{d s_{1}}{s_{1}} \frac{d s_{2}}{s_{2}}
\end{aligned}
$$

and

$$
f_{\lambda, \lambda}^{\prime \prime}(g)=\frac{q^{r+1}-1}{q^{r / 2}} \frac{1}{2 \pi i} \int_{|s|=1} s^{r} \check{\Theta}_{S t, \lambda, s}(g) \frac{d s}{s} .
$$

When $\lambda$ is the trivial character of $\mathfrak{o}^{\times}$(denoted 1 for convenience), the functions $f_{\lambda, \lambda}, f_{\lambda, \lambda}^{\prime}$ and $f_{\lambda, \lambda}^{\prime \prime}$ will be denoted by $f_{1}, f_{1}^{\prime}$ and $f_{1}^{\prime \prime}$ respectively. Since for all $g \in G$ we have $\check{\Theta}_{\lambda, \lambda, s_{1}, s_{2}}(g)=\lambda(\operatorname{det} g)^{-1} \cdot \check{\Theta}_{1,1, s_{1}, s_{2}}(g)$ and $\check{\Theta}_{S t, \lambda, s}(g)=\lambda(\operatorname{det} g)^{-1}$. $\check{\Theta}_{S t, s}(g)$, it follows that $f_{\lambda, \lambda}=(\lambda \circ \text { det })^{-1} f_{1}$. Similarly, $f_{\lambda, \lambda}^{\prime}=(\lambda \circ \text { det })^{-1} f_{1}^{\prime}$ and $f_{\lambda, \lambda}^{\prime \prime}=(\lambda \circ \operatorname{det})^{-1} f_{1}^{\prime \prime}$. Therefore, we now turn our attention to computing $f_{1}$ by evaluating $f_{1}^{\prime}$ and $f_{1}^{\prime \prime}$ separately.

We compute $f_{1}^{\prime \prime}$ first. Without loss of generality, we may take the values of our character functions to be zero on nonregular elements. Using $\check{\Theta}_{S t, s}(g)=\check{\Theta}_{S t}(g)$. $s^{-\operatorname{val}(\operatorname{det} g)}$ for all $g \in G$, from equation (4.3) we get

$$
\begin{aligned}
f_{1}^{\prime \prime}(g) & =\frac{q^{r+1}-1}{q^{r / 2}} \frac{1}{2 \pi i} \int_{|s|=1} s^{r} \check{\Theta}_{S t, s}(g) \frac{d s}{s} \\
& =\frac{q^{r+1}-1}{q^{r / 2}} \check{\Theta}_{S t}(g) \frac{1}{2 \pi i} \int_{|s|=1} s^{r-\operatorname{val}(\operatorname{det} g)} \frac{d s}{s} \\
& = \begin{cases}0, & \text { if } \operatorname{val}(\operatorname{det} g) \neq r, \text { and } \\
\frac{q^{r+1}-1}{q^{r / 2}} \check{\Theta}_{S t}(g), & \text { otherwise. }\end{cases}
\end{aligned}
$$

It is well known, and an easy consequence of Theorem 3 (ii) of [10] together with the realization of the Steinberg representation as a quotient of a certain induced representation by the trivial representation, that for regular semisimple $g \in G$ we get

$$
\check{\Theta}_{S t}(g)= \begin{cases}-1, & \text { if } g \text { elliptic, and } \\ \left(\frac{q^{-k_{1}}+q^{-k_{2}}}{\left|\varpi^{k_{1}} o_{1}-\varpi^{k_{2}} o_{2}\right|}\right)-1, & \text { if } g \in{ }^{G}\left(\varpi^{k_{1}} o_{1}, \varpi^{k_{2}} o_{2}\right) \in{ }^{G} A\end{cases}
$$

(here for any $S \subset G$, we denote by ${ }^{G} S$ the set of $G$-conjugates of elements of $S$; recall also that by the notational convention we are following, $k_{1}, k_{2} \in \mathbb{Z}$ and $o_{1}, o_{2} \in \mathfrak{o}^{\times}$ in the above equation). Thus, $f_{1}^{\prime \prime}(g)$ equals 0 if $g$ is singular or val( $\left.\operatorname{det} g\right) \neq r$. If $\operatorname{val}(\operatorname{det} g)=r$ (so $g$ is regular semisimple as $r$ is odd), then $f_{1}^{\prime \prime}(g)$ equals

$$
\begin{cases}-\frac{q^{r+1}-1}{q^{r / 2}}, & \text { if } g \text { elliptic regular, and } \\ \frac{q^{r+1}-1}{q^{r / 2}} \cdot\left(\frac{q^{-k_{1}}+q^{-k_{2}}}{\left|\varpi^{k_{1}} o_{1}-\varpi^{k_{2}} o_{2}\right|}-1\right), & \text { if } \exists\left(\varpi^{k_{1}} o_{1}, \varpi^{k_{2}} o_{2}\right) \in A \cap G\{g\}, \\ & \text { with } k_{1}+k_{2}=r .\end{cases}
$$


Note that since $r$ is odd, if $\operatorname{val}(\operatorname{det} g)=r$, then $g$ is conjugate to a diagonal matrix if and only if $\operatorname{tr} g \notin \mathfrak{p}^{\lceil r / 2\rceil}$.

Therefore we may write for regular semisimple $g$ :

$$
f_{1}^{\prime \prime}(g)= \begin{cases}0, & \text { if } \operatorname{val}(\operatorname{det} g) \neq r, \\ -\frac{q^{r+1}-1}{q^{r / 2}}, & \text { if } \operatorname{val}(\operatorname{det} g)=r \text { and } \operatorname{tr} g \in \mathfrak{p}^{\lceil r / 2\rceil}, \\ \frac{q^{r+1}-1}{q^{r / 2}} \cdot q^{2 \operatorname{val}(\operatorname{tr} g)-r}, & \text { if } \operatorname{val}(\operatorname{det} g)=r \text { and } \operatorname{tr} g \notin \mathfrak{p}^{\lceil r / 2\rceil} .\end{cases}
$$

Now we turn to $f_{1}^{\prime}(g)$. Recall from Theorem 3 (ii) of [10] that for $g \in G$, $\check{\Theta}_{1,1, s_{1}, s_{2}}(g)=0$ unless $g \in G\left\{\left(\varpi^{k_{1}} o_{1}, \varpi^{k_{2}} o_{2}\right)\right\}$ for some $\left(\varpi^{k_{1}} o_{1}, \varpi^{k_{2}} o_{2}\right) \in A$, in which case:

$$
\check{\Theta}_{1,1, s_{1}, s_{2}}(g)=\frac{s_{1}^{-k_{1}} s_{2}^{-k_{2}}+s_{1}^{-k_{2}} s_{2}^{-k_{1}}}{\left|\varpi^{k_{1}} o_{1}-\varpi^{k_{2}} o_{2}\right|} q^{-\left(k_{1}+k_{2}\right) / 2} .
$$

Hence for regular semisimple $g$, equation (4.2) gives that $f_{1}^{\prime}(g)=0$ unless $g$ is conjugate to some $\left(\varpi^{k_{1}} o_{1}, \varpi^{k_{2}} o_{2}\right)$. Therefore, we fix such a $g$ and a corresponding $k_{1}, k_{2}, o_{1}, o_{2}$ from now, until and including equation (4.5) below. Since $\left(\varpi^{k_{1}} o_{1}, \varpi^{k_{2}} o_{2}\right)$ and $\left(\varpi^{k_{2}} o_{2}, \varpi^{k_{1}} o_{1}\right)$ are conjugate, we may and do also assume without loss of generality that $k_{2} \leq k_{1}$. From equation (4.2) and using the change of variables $s_{2}=s s_{1}$ in the second step below:

$$
\begin{aligned}
f_{1}^{\prime}(g)= & \frac{q(q+1)}{2(2 \pi i)^{2}} \cdot \int_{\left|s_{1}\right|=\left|s_{2}\right|=1}\left(\sum_{j=0}^{r} s_{1}^{j} s_{2}^{r-j}\right) \frac{s_{1}^{-k_{1}} s_{2}^{-k_{2}}+s_{1}^{-k_{2}} s_{2}^{-k_{1}}}{\left|\varpi^{k_{1}} o_{1}-\varpi^{k_{2}} o_{2}\right|} \cdot q^{-\left(k_{1}+k_{2}\right) / 2} \\
& \cdot \frac{\left(s_{1}-s_{2}\right)^{2}}{\left(q s_{1}-s_{2}\right)\left(s_{1}-q s_{2}\right)} \frac{d s_{1}}{s_{1}} \frac{d s_{2}}{s_{2}} \\
= & \frac{q^{1-\left(k_{1}+k_{2}\right) / 2}(q+1)}{2(2 \pi i)^{2}} \cdot \int_{\left|s_{1}\right|=|s|=1} s_{1}^{r}\left(\sum_{i=0}^{r} s^{i}\right) \cdot \frac{s_{1}^{-\left(k_{1}+k_{2}\right)}\left(s^{-k_{2}}+s^{-k_{1}}\right)}{\left|\varpi^{k_{1}} o_{1}-\varpi^{k_{2}} o_{2}\right|} \\
& \cdot \frac{(1-s)^{2}}{(q-s)(1-q s)} \frac{d s_{1}}{s_{1}} \frac{d s}{s} .
\end{aligned}
$$

Integrating over $s_{1}$, this is 0 unless $k_{1}+k_{2}=r$, which we assume from now on. Then $r$ being odd, $k_{1} \neq k_{2}$, so $\left|\varpi^{k_{1}} o_{1}-\varpi^{k_{2}} o_{2}\right|=q^{-\min \left(k_{1}, k_{2}\right)}=q^{-k_{2}}$. Thus, we get

$$
\begin{aligned}
f_{1}^{\prime}(g) & =\frac{q^{1-\left(k_{1}-k_{2}\right) / 2}(q+1)}{2(2 \pi i)} \int_{|s|=1}\left(\sum_{j=0}^{r}\left(s^{j-k_{2}}+s^{j-k_{1}}\right)\right) \frac{(1-s)^{2}}{(q-s)(1-q s)} \frac{d s}{s} \\
& =\frac{q^{1-\left(k_{1}-k_{2}\right) / 2}(q+1)}{2} \sum_{j=0}^{r}\left(I_{j-k_{2}}+I_{j-k_{1}}\right),
\end{aligned}
$$

where for all $l \in \mathbb{Z}, I_{l}$ denotes the integral defined in equation (3.6.3) of [6] (also see the computation just after equation (3.6.4) of the same reference).

Using $k_{1}+k_{2}=r$ and the obvious relation $I_{l}=I_{-l}$ for all $l \in \mathbb{Z}$ (so $I_{j-k_{1}}=$ $I_{j-r+k_{2}}=I_{(r-j)-k_{2}}$ for all $\left.j\right)$, we get

$$
\sum_{j=0}^{r} I_{j-k_{1}}=\sum_{j=0}^{r} I_{j-k_{2}}=\sum_{j=-k_{2}}^{k_{1}} I_{j}
$$


SO

$$
f_{1}^{\prime}(g)=q^{1-\left(k_{1}-k_{2}\right) / 2}(q+1) \sum_{j=-k_{2}}^{k_{1}} I_{j}
$$

From equation (3.6.5) of [6] and from [7], we have for all $l \in \mathbb{Z}$,

$$
I_{l}= \begin{cases}-q^{-|l|-1} \frac{q-1}{q+1}, & \text { if } l \neq 0, \text { and } \\ -q^{-|l|-1} \frac{\frac{q-1}{q+1}+\frac{1}{q},}{} & \text { if } l=0 .\end{cases}
$$

Therefore, we get

$$
f_{1}^{\prime}(g)= \begin{cases}-q^{1-\left(k_{1}-k_{2}\right) / 2}(q+1)\left(\frac{q-1}{q+1} \sum_{j=-k_{2}}^{k_{1}} q^{-|j|-1}\right), & \text { if } k_{2}<0 \\ -q^{1-\left(k_{1}-k_{2}\right) / 2}(q+1)\left(\frac{q-1}{q+1} \sum_{j=-k_{2}}^{k_{1}} q^{-|j|-1}-\frac{1}{q}\right), & \text { if } k_{2} \geq 0 .\end{cases}
$$

Simplifying, we get

$$
f_{1}^{\prime}(g)= \begin{cases}-q^{-(r / 2)+k_{2}-k_{1}}\left(q^{r+1}-1\right), & \text { if } \min \left(k_{1}, k_{2}\right)<0, \text { and } \\ q^{-r / 2}\left(1+q^{k_{2}-k_{1}}\right), & \text { if } \min \left(k_{1}, k_{2}\right) \geq 0\end{cases}
$$

We rewrite this as

$$
f_{1}^{\prime}(g)= \begin{cases}-q^{2 \operatorname{val}(\operatorname{tr} g)-r} \cdot \frac{q^{r+1}-1}{q^{r / 2}}, & \text { if } \operatorname{val}(\operatorname{tr} g)<0, \text { and } \\ \frac{1+q^{2 \operatorname{val}(\operatorname{tr} g)-r}}{q^{r / 2}}, & \text { if } 0 \leq \operatorname{val}(\operatorname{tr} g) \leq\lfloor r / 2\rfloor .\end{cases}
$$

Recalling that $f_{1}=f_{1}^{\prime}+f_{1}^{\prime \prime}$, we get that for an arbitrary regular semisimple $g \in G, f_{1}(g)=0$ unless $\operatorname{val}(\operatorname{det} g)=r$, in which case we have

$$
f_{1}(g)= \begin{cases}-\frac{q^{r+1}-1}{q^{r / 2}}, & \text { if } \operatorname{val}(\operatorname{tr} g) \geq\lceil r / 2\rceil \\ \frac{q^{2 \operatorname{val}(\operatorname{tr} g)+1}+1}{q^{r / 2}}, & \text { if } 0 \leq \operatorname{val}(\operatorname{tr} g) \leq\lfloor r / 2\rfloor \\ 0, & \text { if } \operatorname{val}(\operatorname{tr} g)<0\end{cases}
$$

Now we turn to $f_{\lambda, \lambda} * \operatorname{ch}_{K_{B}}$, for a given character $\lambda$ of $\mathfrak{o}^{\times}$with $\lambda^{r}=1$. Since $r$ is relatively prime to $q$ and since $\lambda^{r}=1, \lambda$ has conductor 1 , and hence by (ii) of Hypothesis 2.1] $\lambda(\operatorname{det} 1+x)=1$ for all $x \in B$. Thus, we have

$$
\int_{B} f_{\lambda, \lambda}\left(g_{x}\right) d x=\lambda(\operatorname{det} g)^{-1} \int_{B} f_{1}\left(g_{x}\right) d x
$$

(see Notation 2.3) and it is the integral

$$
\int_{B} f_{1}\left(g_{x}\right) d x
$$

that we will be concerned with.

First, this integral is clearly 0 if $\operatorname{val}(\operatorname{det} g) \neq r$, so assume this to be the case. Then we have three cases: 
(i) Suppose $\min \left(k_{B, g},\lceil r / 2\rceil\right)>\operatorname{val}(\operatorname{tr} g)$. Then by equation (4.6) $f_{1}\left(g_{x}\right)=$ $f_{1}(g)$ for all $x \in B$, so the above integral equals $f_{1}(g)$, which in turn equals $\left(q^{2 \operatorname{val}(\operatorname{tr} g)+1}+1\right) q^{-r / 2}$ if $0 \leq \operatorname{val}(\operatorname{tr} g) \leq\lfloor r / 2\rfloor$, and 0 if $\operatorname{val}(\operatorname{tr} g)<0$.

(ii) Suppose $\min \left(\operatorname{val}(\operatorname{tr} g), k_{B, g}\right) \geq\lceil r / 2\rceil$. Then for all $x \in B, \operatorname{val}\left(\operatorname{tr} g_{x}\right) \geq$ $\lceil r / 2\rceil$, so clearly $f_{1}(g)$ as well as the integral (4.8) equals $-\frac{q^{r+1}-1}{q^{r / 2}}$.

(iii) Suppose $\min (\operatorname{val}(\operatorname{tr} g),\lceil r / 2\rceil) \geq k_{B, g}$. Since $\operatorname{val}(\operatorname{tr} g) \geq k_{B, g}$, the map

$$
\tau: x \mapsto \operatorname{tr}\left(g_{x}\right)
$$

is a surjection from $B$ to $\mathfrak{p}^{k_{B, g}}$ and is, moreover, the composition of a translation in $\mathfrak{p}^{k_{B, g}}$ and a homomorphism of topological groups. Therefore, if $\mathfrak{p}^{k_{B, g}}$ is given the normalized Haar measure, then for any measurable $U \subset \mathfrak{p}^{k_{B, g}}, \tau^{-1}(U) \subset B$ has the same measure as $U$. Therefore, if for the time being $\mu_{B}$ denotes our normalized Haar measure on $B$ then

$$
\forall j \text { with } k_{B, g} \leq j \leq\lfloor r / 2\rfloor, \mu_{B}\left((\operatorname{val} \circ \tau)^{-1}(j)\right)=\frac{q-1}{q^{j-k_{B, g}+1}},
$$

and

$$
\mu_{B}\left((\operatorname{val} \circ \tau)^{-1}(\lfloor r / 2\rfloor, \infty)\right)=\frac{1}{q^{\lceil r / 2\rceil-k_{B, g}}} .
$$

Therefore integral (4.8) is equal to

$$
\left(\sum_{j=\max \left(0, k_{B, g}\right)}^{\lfloor r / 2\rfloor}\left(\frac{q^{2 j+1}+1}{q^{r / 2}}\right) \cdot \frac{q-1}{q^{j-k_{B, g}+1}}\right)-\left(\frac{q^{r+1}-1}{q^{r / 2}}\right) \cdot \frac{1}{q^{\lceil r / 2\rceil-k_{B, g}}},
$$

which, setting $j_{0}=\max \left(0, k_{B, g}\right)$ for convenience, is equal to

$$
\frac{1}{q^{r / 2-k_{B, g}}} \cdot\left(\sum_{j=j_{0}}^{\lfloor r / 2\rfloor}\left(\left(q^{j+1}-q^{-j-1}\right)-\left(q^{j}-q^{-j}\right)\right)\right)-\frac{1}{q^{r / 2-k_{B, g}}} \cdot\left(q^{\lceil r / 2\rceil}-q^{-\lceil r / 2\rceil}\right),
$$

which clearly simplifies to $-q^{-r / 2+k_{B, g}}\left(q^{j_{0}}-q^{-j_{0}}\right)$, which equals 0 if $k_{B, g} \leq$ 0 and $-q^{-r / 2}\left(q^{2 k_{B, g}}-1\right)$ otherwise.

Set $\chi_{r}(g)$ to be 1 if $\operatorname{det} g \in\left(F^{\times}\right)^{r}$ and 0 otherwise. Then we have from equations (2.5) and (4.7) that $f_{B}^{(0)}(g)$ equals 0 if $\operatorname{val}(\operatorname{det} g) \neq r$, and for $g$ with $\operatorname{val}(\operatorname{det} g)=r$ it is given by

$$
f_{B}^{(0)}(g)= \begin{cases}0, & \text { if } \operatorname{tr} g \notin \mathfrak{o} \text { or } k_{B, g} \leq 0, \\ \chi_{r}(g)(q-1, r) \cdot \frac{q^{2 \operatorname{val}(\operatorname{tr} g)+1}+1}{q^{r / 2}}, & \text { if } 0 \leq \operatorname{val}(\operatorname{tr} g)<\min \left(k_{B, g},\lceil r / 2\rceil\right), \\ -\chi_{r}(g)(q-1, r) \cdot \frac{q^{r+1}-1}{q^{r / 2}}, & \text { if }\lceil r / 2\rceil \leq \min \left(\operatorname{val}(\operatorname{tr} g), k_{B, g}\right), \\ -\chi_{r}(g)(q-1, r) \cdot \frac{q^{2 k_{B, g}}-1}{q^{r / 2}}, & \text { if } 0 \leq k_{B, g} \leq \min (\operatorname{val}(\operatorname{tr} g),\lfloor r / 2\rfloor) .\end{cases}
$$

To interpret the above equation, recall that for a character $\lambda$ of $\mathfrak{o}^{\times}$, we view it as a character of $F^{\times}$through $x \mapsto x / \varpi^{\text {val } x}$. Also, in the above equation there is some overlap between the cases involved, but the corresponding formulas are clearly compatible. 


\section{RAMIFIED PRINCIPAL SERIES COMPONENTS}

Let $\mu, \nu$ be characters of $\mathfrak{o}^{\times}$, with $\mu \neq \nu$. Let $a, b \in \mathbb{N} \cup\{0\}$ with $a>b$, such that $a+b=r$. Recall that $z_{\mu, \nu, a, b} \in \mathcal{Z}$, realized by the locally integrable function $f_{\mu, \nu, a, b}$, acts each of the induced representations $\operatorname{Ind}_{B}^{G}\left(\mu s_{1}^{\operatorname{val}(\cdot)}, \nu s_{2}^{\operatorname{val}(\cdot)}\right)$ by $s_{1}^{a} s_{2}^{b}$ and by 0 on representations outside this Bernstein component.

Recall also that this Bernstein component can be identified with $\mathbb{C}^{\times} \times \mathbb{C}^{\times}$, with $\left(s_{1}, s_{2}\right)$ corresponding to the conjugacy class of the character

$$
\left(\mu s_{1}^{\operatorname{val}(\cdot)}, \nu s_{2}^{\operatorname{val}(\cdot)}\right):\left(\varpi^{k_{1}} o_{1}, \varpi^{k_{2}} o_{2}\right) \mapsto \mu\left(o_{1}\right) \nu\left(o_{2}\right) s_{1}^{k_{1}} s_{2}^{k_{2}},
$$

of $A$. In this case, since $\mu \neq \nu$, by Theorem 4.3 of [1] the Plancherel measure is constant on representations with cuspidal support in $\Omega$, and is given by

$$
\mu\left(s_{1}, s_{2}\right)=\left(\frac{q+1}{q}\right)^{2} q^{f\left(\mu^{-1} \nu\right)},
$$

(recall Notation 2.4 $f\left(\mu^{-1} \nu\right)$ denotes the conductor of $\left.\mu^{-1} \nu\right)$. Henceforth, for any character $\lambda$ of $\mathfrak{o}^{\times}, f(\lambda)$ will denote the conductor of $\lambda$. Write $\Theta_{\mu, \nu, s_{1}, s_{2}}$ for the character of $\pi_{\mu, \nu, s_{1}, s_{2}}$ and $\check{\Theta}_{\mu, \nu, s_{1}, s_{2}}(g)$ for $\Theta_{\mu, \nu, s_{1}, s_{2}}\left(g^{-1}\right)$. If $\varphi \in C_{c}^{\infty}(G)$, following the inversion formula cited around equation (4.1),

$$
\begin{aligned}
z_{\mu, \nu, a, b}(\varphi)=c(G \mid A)^{-2} \gamma(G \mid A)^{-1} \cdot \frac{1}{1} \cdot\left(\frac{1}{2 \pi i}\right)^{2} \int_{\left|s_{1}\right|=\left|s_{2}\right|=1} z_{\mu, \nu, a, b}\left(\pi_{\mu, \nu, s_{1}, s_{2}}\right) \\
\cdot \mu_{G \mid A}\left(\mu s_{1}^{\operatorname{val}(\cdot)}, \nu s_{2}^{\operatorname{val}(\cdot)}\right) d\left(\mu s_{1}^{\operatorname{val}(\cdot)}, \nu s_{2}^{\operatorname{val}(\cdot)}\right) \cdot \check{\Theta}_{\mu, \nu, s_{1}, s_{2}}(\varphi) \frac{d s_{1}}{s_{1}} \frac{d s_{2}}{s_{2}} \\
=\frac{q}{(q+1)}\left(\frac{1}{2 \pi i}\right)^{2} \int_{\left|s_{1}\right|=\left|s_{2}\right|=1} s_{1}^{a} s_{2}^{b} \cdot\left(\frac{q+1}{q}\right)^{2} q^{f\left(\mu^{-1} \nu\right)} \\
\cdot 1 \cdot\left(\int_{G} \varphi(g) \check{\Theta}_{\mu, \nu, s_{1}, s_{2}}(g) d g\right) \frac{d s_{1}}{s_{1}} \frac{d s_{2}}{s_{2}} \\
=\int_{G} \varphi(g)\left(\frac{q+1}{q} q^{f\left(\mu^{-1} \nu\right)}\left(\frac{1}{2 \pi i}\right)^{2} \int_{\left|s_{1}\right|=\left|s_{2}\right|=1} s_{1}^{a} s_{2}^{b} \check{\Theta}_{\mu, \nu, s_{1}, s_{2}}(g) \frac{d s_{1}}{s_{1}} \frac{d s_{2}}{s_{2}}\right) d g,
\end{aligned}
$$

by Fubini's theorem. Therefore, for $g \in G$,

$$
f_{\mu, \nu, a, b}(g)=(q+1) q^{f\left(\mu^{-1} \nu\right)-1}\left(\frac{1}{2 \pi i}\right)^{2} \int_{\left|s_{1}\right|=\left|s_{2}\right|=1} s_{1}^{a} s_{2}^{b} \check{\Theta}_{\mu, \nu, s_{1}, s_{2}}(g) \frac{d s_{1}}{s_{1}} \frac{d s_{2}}{s_{2}} .
$$

In particular, $f_{\mu, \nu, a, b}$ is supported on regular elements in ${ }^{G} A$, the set of elements of $G$ conjugate to some element of $A$. For $g$ conjugate to $\left(\varpi^{k_{1}} o_{1}, \varpi^{k_{2}} o_{2}\right)$, by Theorem 3 (ii) of [10,

$$
\check{\Theta}_{\mu, \nu, s_{1}, s_{2}}(g)=\frac{s_{1}^{-k_{1}} \mu\left(o_{1}^{-1}\right) s_{2}^{-k_{2}} \nu\left(o_{1}^{-1}\right)+s_{2}^{-k_{1}} \nu\left(o_{1}^{-1}\right) s_{1}^{-k_{2}} \mu\left(o_{2}^{-1}\right)}{\left|\varpi^{k_{1}} o_{1}-\varpi^{k_{2}} o_{2}\right|} \cdot q^{-\left(k_{1}+k_{2}\right) / 2} .
$$

Therefore,

$$
\begin{aligned}
f_{\mu, \nu, a, b}(g)= & \frac{(q+1) q^{f\left(\mu^{-1} \nu\right)-1-\left(k_{1}+k_{2}\right) / 2}}{(2 \pi i)^{2}} \int_{\left|s_{1}\right|=\left|s_{2}\right|=1} \\
& \frac{s_{1}^{a-k_{1}} s_{2}^{b-k_{2}} \mu\left(o_{1}^{-1}\right) \nu\left(o_{2}^{-1}\right)+s_{1}^{a-k_{2}} s_{2}^{b-k_{1}} \mu\left(o_{2}^{-1}\right) \nu\left(o_{1}^{-1}\right)}{\left|\varpi^{k_{1}} o_{1}-\varpi^{k_{2}} o_{2}\right|} \frac{d s_{1}}{s_{1}} \frac{d s_{2}}{s_{2}} .
\end{aligned}
$$


This is zero unless $\left(k_{1}, k_{2}\right)$ is either $(a, b)$ or $(b, a)$ (these two are "Weyl-conjugate" cases). From now we assume this to be the case, so let $g$ be conjugate to some $\left(\varpi^{a} o_{1}, \varpi^{b} o_{2}\right)$, so $k_{1}=a, k_{2}=b$. In this case, $k_{1} \neq k_{2}$ as $a+b=r$ is odd, so that $\left|\varpi^{k_{1}} o_{1}-\varpi^{k_{2}} o_{2}\right|=q^{-\min \left(k_{1}, k_{2}\right)}=q^{-b}$. Therefore,

$$
f_{\mu, \nu, a, b}(g)=(q+1) q^{f\left(\mu^{-1} \nu\right)-1-(a-b) / 2} \mu\left(o_{1}^{-1}\right) \nu\left(o_{2}^{-1}\right)=c_{\mu, \nu, a, b} \cdot \mu\left(o_{1}^{-1}\right) \nu\left(o_{2}^{-1}\right),
$$

where for convenience we have written $c_{\mu, \nu, a, b}$ for $(q+1) q^{f\left(\mu^{-1} \nu\right)-1-(a-b) / 2}$.

We once again take $B$ to be a lattice in $\mathbf{M}_{2}(F)$ satisfying Hypothesis 2.1, and wish to find, for $g \in G$,

$$
\int_{B} f_{\mu, \nu, a, b}\left(g_{x}\right) d x
$$

Hypothesis 2.1 (i) gives that, for $g$ conjugate to some $\left(\varpi^{a} o_{1}, \varpi^{b} o_{2}\right)$ we have

$$
b+m_{B} \geq k_{B, g} .
$$

Lemma 5.1. For all $g \in G$,

$$
\int_{B} f_{\mu, \nu, a, b}\left(g_{x}\right) d x= \begin{cases}f_{\mu, \nu, a, b}(g), & \text { if } f(\mu) \leq m_{B} \text { and } f\left(\mu^{-1} \nu\right) \leq k_{B, g}-b, \text { and } \\ 0, & \text { otherwise. }\end{cases}
$$

Proof. Suppose $g \in G$ lies in the support of $f_{\mu, \nu, a, b}$, i.e., val tr $g=b$ and val $\operatorname{det} g=$ $r$. Note that if $f\left(\mu^{-1} \nu\right) \leq k_{B, g}-b$, it means $k_{B, g}>b$ (as we are assuming $\mu \neq \nu$ ), so for all $x \in B$ we have, apart from valdet $g_{x}=r$, also that $\operatorname{tr} g_{x}=b$ and $k_{B, g_{x}}=k_{B, g}$. This means that we may only need to prove the assertion of the lemma for those $g \in G$ such that val $\operatorname{tr} g=b$ and val $\operatorname{det} g=r$.

Moreover, in the case where $m_{B}<f(\mu)$ or $k_{B, g}<f\left(\mu^{-1} \nu\right)+b$, since $k_{B, g}$ is invariant under right multiplication by $1+B$, we may if necessary multiply $B$ by $\varpi^{m}$ for some $m \in \mathbb{N}$ (this increases both $m_{B}$ and $k_{B, g}$ by $m$ and respects Hypothesis 2.1), to ensure that $m_{B} \geq f(\mu)-1$ and $k_{B, g} \geq f\left(\mu^{-1} \nu\right)+b-1$, with at least one of these two being an equality. Recall that for $g \in G$ conjugate to an element of the form $t=\left(\varpi^{a} o_{1}, \varpi^{b} o_{2}\right)$ with $o_{1}, o_{2} \in \mathfrak{o}^{\times}$we have

$$
f_{\mu, \nu, a, b}(g)=c_{\mu, \nu, a, b} \cdot \mu\left(o_{1}^{-1}\right) \nu\left(o_{2}^{-1}\right) .
$$

Fix such a $g$ for the time being, and pick $\left(\lambda_{1}, \lambda_{2}\right) \in A$ conjugate to $g$, with $\operatorname{val}\left(\lambda_{1}\right)=$ $a$ and $\operatorname{val}\left(\lambda_{2}\right)=b$. Note that for all $x \in B, f_{\mu, \nu, a, b}\left(g_{x}\right)$ equals 0 unless $g_{x}$ is conjugate to some $\left(\lambda_{1} o_{1}, \lambda_{2} o_{2}\right)$ with $o_{1}, o_{2} \in \mathfrak{o}^{\times}$, in which case

$$
f_{\mu, \nu, a, b}\left(g_{x}\right)=f_{\mu, \nu, a, b}(g) \cdot \mu^{-1}\left(o_{1} o_{2}\right) \cdot\left(\nu^{-1} \mu\right)\left(o_{2}\right) .
$$

Our key step will be to prove that if $g_{x}$, for some $x \in B$, is conjugate to an element of the form $\left(\lambda_{1} o_{1}, \lambda_{2} O_{2}\right)$ with $o_{1}, o_{2} \in \mathfrak{o}^{\times}$, then

$$
\left(\nu^{-1} \mu\right)\left(o_{2}\right)=\left(\nu^{-1} \mu\right)\left(1+\frac{\operatorname{tr} g x}{\lambda_{2}}\right) .
$$

To prove this, suppose $o_{1}, o_{2} \in \mathfrak{o}^{\times}$and $x \in B$ are such that $\left(\lambda_{1} o_{1}, \lambda_{2} o_{2}\right)$ is conjugate to $g_{x}$. Then $\lambda_{1} o_{1}+\lambda_{2} O_{2} \in \operatorname{tr} g+\mathfrak{p}^{k_{B, g}}$, or,

$$
\lambda_{1}\left(o_{1}-1\right)+\lambda_{2}\left(o_{2}-1\right) \in \mathfrak{p}^{k_{B, g}} .
$$

Furthermore, $o_{1} o_{2}=\operatorname{det}(1+x) \in 1+\mathfrak{p}^{m_{B}}$, so the observation

$$
\operatorname{val}\left(o_{1}-1\right)=\operatorname{val}\left(o_{1} o_{2}-o_{2}\right)=\operatorname{val}\left(\left(o_{1} o_{2}-1\right)-\left(o_{2}-1\right)\right)
$$

shows that either both $\operatorname{val}\left(o_{1}-1\right), \operatorname{val}\left(o_{2}-1\right) \geq m_{B}$ or $\operatorname{val}\left(o_{1}-1\right)=\operatorname{val}\left(o_{2}-\right.$ $1)$. We claim that in either case $\operatorname{val}\left(o_{1}-1\right) \geq f\left(\mu^{-1} \nu\right)-1$. In the former case, 
$\operatorname{val}\left(o_{1}-1\right), \operatorname{val}\left(o_{2}-1\right) \geq m_{B}$, which by equation (5.2), is greater than or equal to $k_{B, g}-b$, which by our assumption is greater than or equal to $f\left(\mu^{-1} \nu\right)-1$. In the latter case, since $\operatorname{val}\left(\lambda_{1}\right)=a \neq b=\operatorname{val}\left(\lambda_{2}\right)$, we see from equation (5.5) that $\operatorname{val}\left(o_{2}-1\right)+b \geq k_{B, g} \geq b+f\left(\mu^{-1} \nu\right)-1$, so $\operatorname{val}\left(o_{2}-1\right) \geq f\left(\mu^{-1} \nu\right)-1$, so $\operatorname{val}\left(o_{1}-1\right)=\operatorname{val}\left(o_{2}-1\right) \geq f\left(\mu^{-1} \nu\right)-1$ as well.

Therefore, in either case we have

$$
\left(o_{2}^{-1} \cdot\left(1+\operatorname{tr} \frac{g x}{\lambda_{2}}\right)\right)-1=o_{2}^{-1}\left(\frac{\lambda_{1}\left(o_{1}-1\right)}{\lambda_{2}}\right) \in \mathfrak{p}^{f\left(\mu^{-1} \nu\right)-1+a-b} \subset \mathfrak{p}^{f\left(\mu^{-1} \nu\right)},
$$

as $a>b$. This gives equation (5.4).

Using (ii) of Hypothesis 2.1 and our assumption that $m_{B} \geq f(\mu)-1$, we get

$$
\forall x \in B, \mu(\operatorname{det} 1+x)=\mu(1+\operatorname{tr} x+\operatorname{det} x)=\mu(1+\operatorname{tr} x) .
$$

That $m_{B} \geq f(\mu)-1$, also shows that $x \mapsto \mu(1+\operatorname{tr} x)=\mu(\operatorname{det} 1+x)$ is a character on $B$.

Since we have assumed $m_{B} \geq f(\mu)-1$ and $k_{B, g} \geq f\left(\mu^{-1} \nu\right)+b-1$, it is enough to consider four cases: (i) $m_{B}>f(\mu)-1$ and $k_{B, g}>f\left(\mu^{-1} \nu\right)+b-1$; (ii) $m_{B}=f(\mu)-1$; (iii) $m_{B}>f(\mu)-1$ and $k_{B, g}=f\left(\mu^{-1} \nu\right)+b-1>b$; and (iv) $m_{B}>f(\mu)-1$ and $k_{B, g}=f\left(\mu^{-1} \nu\right)+b-1=b$. Here are these cases treated one by one:

(i) Suppose $m_{B}>f(\mu)-1$ and $k_{B, g}>f\left(\mu^{-1} \nu\right)+b-1$. Then using equation (5.6) and that $m_{B} \geq f(\mu), \mu(\operatorname{det} 1+x)=1$ for all $x \in B$. Further, for all $x \in B, \operatorname{val}(\operatorname{tr} g x) \geq k_{B, g} \geq b+f\left(\mu^{-1} \nu\right)>b$, so $g_{x}$ is indeed conjugate to an element of the form $\left(\lambda_{1} o_{1}, \lambda_{2} o_{2}\right)$ with $o_{1}, o_{2} \in \mathfrak{o}^{\times}$. This with equation (5.4) and $\operatorname{val}(\operatorname{tr} g x) \geq b+f\left(\mu^{-1} \nu\right)$ gives that $f_{\mu, \nu, a, b}\left(g_{x}\right)=f_{\mu, \nu, a, b}(g)$ for all $x \in B$. Therefore,

$$
\int_{B} f_{\mu, \nu, a, b}\left(g_{x}\right) d x=f_{\mu, \nu, a, b}(g) .
$$

(ii) Suppose $m_{B}=f(\mu)-1$. Let us first see why it is enough to prove that there exists $x_{0} \in B$ such that $\operatorname{tr} x_{0} \in \mathfrak{p}^{m_{B}} \backslash \mathfrak{p}^{m_{B}+1}$ and $\operatorname{tr} \lambda_{2}^{-1} g x_{0} \in \mathfrak{p}^{f\left(\mu^{-1} \nu\right)}$. First, this would imply $\operatorname{tr} g x_{0} \in \mathfrak{p}^{b+f\left(\mu^{-1} \nu\right)} \subset \mathfrak{p}^{b+1}$ so that for all $x \in B$, $g_{x}$ would be conjugate to some $\left(\lambda_{1} o_{1}, \lambda_{2} o_{2}\right)$ with $o_{1}, o_{2} \in \mathfrak{o}^{\times}$if and only if $g_{x+x_{0}}$ satisfied the same property. This together with equation (5.4) would give that, for all $x \in B$,

$$
f_{\mu, \nu, a, b}\left(g_{x+x_{0}}\right)=\mu^{-1}\left(\operatorname{det} 1+x_{0}\right) f_{\mu, \nu, a, b}\left(g_{x}\right) .
$$

Then, changing variables $x \mapsto x+x_{0}$, we would get that

$$
\int_{B} f\left(g_{x}\right) d x=\mu^{-1}\left(\operatorname{det} 1+x_{0}\right) \int_{B} f\left(g_{x}\right) d x=\mu^{-1}\left(1+\operatorname{tr} x_{0}\right) \int_{B} f\left(g_{x}\right) d x
$$

by equation (5.6) and since we may assume $\mu\left(1+\operatorname{tr} x_{0}\right) \neq 1$ (because $\left.\operatorname{val}\left(\operatorname{tr} x_{0}\right) \in \mathfrak{p}^{m_{B}} \backslash \mathfrak{p}^{m_{B}+1}=\mathfrak{p}^{f(\mu)-1} \backslash \mathfrak{p}^{f(\mu)}\right)$, this integral would vanish.

Thus, it is enough to prove that there exists $x_{0} \in B$ with $\operatorname{tr} x_{0} \in \mathfrak{p}^{m_{B}} \backslash$ $\mathfrak{p}^{m_{B}+1}$ and $\operatorname{tr} \lambda_{2}^{-1} g x_{0} \in \mathfrak{p}^{f\left(\mu^{-1} \nu\right)}$. Since $x \mapsto \operatorname{tr} \lambda_{2}^{-1} g x$ is a map from $B$ onto $\mathfrak{p}^{k_{B, g}-b}$, this is clear if $k_{B, g}-b \geq f\left(\mu^{-1} \nu\right)$, so for the rest of this paragraph we assume $k_{B, g}-b<f\left(\mu^{-1} \nu\right)$ so that $f\left(\mu^{-1} \nu\right)=k_{B, g}-b+1$. To find 
the required $x_{0}$, it is enough to prove that the homomorphism from $B$ to $\left(\mathfrak{p}^{m_{B}} / \mathfrak{p}^{m_{B}+1}\right) \times\left(\mathfrak{p}^{m_{B}} / \mathfrak{p}^{m_{B}+1}\right)$ induced by

$$
x \mapsto\left(\operatorname{tr} x, \operatorname{tr} \varpi^{m_{B}+b-k_{B, g}} \lambda_{2}^{-1} g x\right)
$$

is surjective. If this were not the case, there would exist $c_{1} \in \mathfrak{o}^{\times}$such that, for all $x \in B$,

$$
\operatorname{tr}\left(\left(c_{1} \varpi^{m_{B}+b-k_{B, g}} \lambda_{2}^{-1} g-I_{2}\right) x\right) \in \mathfrak{p}^{m_{B}+1},
$$

where $I_{2}$ denotes the identity element in $G$. This means that

$$
c_{1} \varpi^{m_{B}+b-k_{B, g}} \lambda_{2}^{-1} g-I_{2} \in \varpi^{m_{B}} B^{\perp} .
$$

So, for some $c \in \mathfrak{o}^{\times}$,

$$
g \in \varpi^{k_{B, g}-m_{B}} c\left(I_{2}+\varpi^{m_{B}} B^{\perp}\right) .
$$

This together with (iii) of Hypothesis 2.1 gives val det $g=2\left(k_{B, g}-m_{B}\right)$ which is a contradiction as val $\operatorname{det} g=r$ is odd.

(iii) Suppose $f(\mu) \leq m_{B}$ and $f\left(\mu^{-1} \nu\right)=k_{B, g}-b+1>1$. First, since $f(\mu) \leq m_{B}$ equation (5.6) gives that $x \mapsto \mu(\operatorname{det} 1+x)$ is trivial on $B$. Further, since $k_{B, g}>b$, we have that for all $x \in B$, val tr $g_{x}=b$ and val $\operatorname{det} g_{x}=a+b$. Thus, for any such $x$ we have from equations (5.3) and (5.4):

$$
\begin{aligned}
f_{\mu, \nu, a, b}\left(g_{x}\right) & =f_{\mu, \nu, a, b}(g) \cdot\left(\mu^{-1}\right)(\operatorname{det} 1+x) \cdot\left(\nu^{-1} \mu\right)\left(1+\frac{\operatorname{tr} g x}{\lambda_{2}}\right) \\
& =f_{\mu, \nu, a, b}(g) \cdot\left(\nu^{-1} \mu\right)\left(1+\frac{\operatorname{tr} g x}{\lambda_{2}}\right) .
\end{aligned}
$$

Now for $x \in B$, the value of $\left(\nu^{-1} \mu\right)\left(1+\lambda_{2}^{-1} \operatorname{tr} g x\right)$ depends only on the image of $1+\lambda_{2}^{-1} \operatorname{tr} g x$ in $\left(1+\mathfrak{p}^{f\left(\mu^{-1} \nu\right)-1}\right) / 1+\mathfrak{p}^{f\left(\mu^{-1} \nu\right)} \cong \mathfrak{p}^{f\left(\mu^{-1} \nu\right)-1} / \mathfrak{p}^{f\left(\mu^{-1} \nu\right)}$. This together with $x \mapsto \lambda_{2}^{-1} \operatorname{tr} g x$ being a surjection from $B$ onto $\mathfrak{p}^{k_{B, g}-b}=$ $\mathfrak{p}^{f\left(\mu^{-1} \nu\right)-1}$ gives that $x \mapsto\left(\nu^{-1} \mu\right)\left(1+\lambda_{2}^{-1} \operatorname{tr} g x\right)$ is a nontrivial character, call it $\Lambda$, on $B$. Thus,

$$
\int_{B} f_{\mu, \nu, a, b}\left(g_{x}\right) d x=f_{\mu, \nu, a, b}(g) \cdot \int_{B} \Lambda(x) d x=0 .
$$

(iv) Suppose $f(\mu) \leq m_{B}$, and $f\left(\mu^{-1} \nu\right)=k_{B, g}-b+1=1$. We are assuming $f(\mu) \leq m_{B}, x \mapsto \mu(\operatorname{det} 1+x)$ is trivial on $B$ exactly as in the previous case. For all $x \in B$, we still have val $\operatorname{det} g_{x}=a+b$, but we only have in general that valtr $g x \in \mathfrak{p}^{k_{B, g}}=\mathfrak{p}^{b}$, and thus $\operatorname{val}\left(\operatorname{tr} g_{x}\right)=b$ if and only if $\operatorname{tr} g x \not \equiv-\operatorname{tr} g \bmod \mathfrak{p}^{b} \equiv-\lambda_{2} \bmod \mathfrak{p}^{b}$ (here we are using that $a>b$ ). This last condition is true if and only if $1+\lambda_{2}^{-1} \operatorname{tr} g x \notin \mathfrak{p}$. Thus we have

$f_{\mu, \nu, a, b}\left(g_{x}\right)= \begin{cases}f_{\mu, \nu, a, b}(g) \cdot\left(\nu^{-1} \mu\right)\left(1+\frac{\operatorname{tr} g x}{\lambda_{2}}\right), & \text { if } 1+\frac{\operatorname{tr} g x}{\lambda_{2}} \notin \mathfrak{p}, \text { and } \\ 0, & \text { otherwise. }\end{cases}$

Note that for each $x \in B,\left(\nu^{-1} \mu\right)\left(1+\lambda_{2}^{-1} \operatorname{tr} g x\right)$ depends only on the image of $1+\lambda_{2}^{-1}(\operatorname{tr} g x)$ in $\mathfrak{o} / \mathfrak{p}$. But given $\alpha \in \mathfrak{o} / \mathfrak{p}$, the measure of the set of all $x$ in $B$ such that $1+\lambda_{2}^{-1} \operatorname{tr} g x$ has image $\alpha$ in $\mathfrak{o} / \mathfrak{p}$ is independent of $\alpha$, since the map $x \mapsto \lambda_{2}^{-1} \operatorname{tr} g x$ is a homomorphism from $B$ onto $\mathfrak{o}$. Since $\nu^{-1} \mu$ 
induces a nontrivial character $\overline{\nu^{-1} \mu}$ of $\mathbb{F}_{q}^{\times}$(we are assuming $\mu \neq \nu$ ), we conclude that

$$
\int_{B} f_{\mu, \nu, a, b}\left(g_{x}\right) d x=(\text { constant }) \cdot \sum_{\left.c \in \mathbb{F}_{q}^{\times}\right)} \overline{\nu^{-1} \mu}(c)=0 .
$$

By Lemma 5.1, for fixed $a, b$ with $a+b=r$ and $a>b$, and for $g \in G$ conjugate to some $\left(\varpi^{a} o_{1}, \varpi^{b} o_{2}\right)$, we have

$$
f_{B}^{(>0, b)}(g)=\sum_{\substack{\mu \neq \nu \\ f(\mu) \leq m_{B} \\ f\left(\mu^{-1} \nu\right) \leq k_{B, g}-b \\ \mu^{a} \nu^{b}=1}}(q+1) q^{f\left(\mu^{-1} \nu\right)-1-(a-b) / 2} \mu\left(o_{1}^{-1}\right) \nu\left(o_{2}^{-1}\right) .
$$

For $n \in \mathbb{N}$ and $g \in G$, set

$$
f_{B}^{(n, b)}(g)=\sum_{\substack{f(\mu) \leq m_{B} \\ f\left(\mu^{-1} \nu\right)=n \\ \mu^{a} \nu^{b}=1}}\left(f_{\mu, \nu, a, b} * \operatorname{ch}_{K_{B}}\right)(g)
$$

which by equation (5.1) and Lemma 5.1 equals

$$
\sum_{\substack{f(\mu) \leq m_{B} \\ f\left(\mu^{-1} \nu\right)=n \leq k_{B, g}-b \\ \mu^{a} \nu^{b}=1}}(q+1) q^{f\left(\mu^{-1} \nu\right)-1-(a-b) / 2} \mu\left(o_{1}^{-1}\right) \nu\left(o_{2}^{-1}\right)
$$

if $g$ is conjugate to some $\left(\varpi^{a} o_{1}, \varpi^{b} o_{2}\right)$, and 0 otherwise. Moreover, we have

$$
f_{B}^{(>0)}(g)=\sum_{\substack{a+b=r \\ b<a}} \sum_{\substack{n \in \mathbb{N} \\ n \leq k_{B}, g}} f_{B}^{(n, b)}(g)
$$

In what follows, we will use that, since $r<p$, the equation $\mu^{a} \nu^{b}=1$ and the resulting equations $\left(\mu^{-1} \nu\right)^{a}=\nu^{r}$ and $\left(\mu^{-1} \nu\right)^{b}=\mu^{-r}$ give that, if $f\left(\mu^{-1} \nu\right) \geq 1$, then $f(\mu) \leq f\left(\mu^{-1} \nu\right)$ and $f(\nu) \leq f\left(\mu^{-1} \nu\right)$.

It is clear that $f_{B}^{(>0)}(g)=0$ if $g$ is not conjugate to any $\left(\varpi^{a} o_{1}, \varpi^{b} o_{2}\right)$ with $a+b=r$. Hence, for the rest of this section we assume $g$ to be conjugate to some fixed $\left(\varpi^{a} o_{1}, \varpi^{b} o_{2}\right)$, and proceed to compute $f_{B}^{(>0)}(g)=f_{B}^{(>0, b)}(g)$. By equation (5.2), we have for $k_{B, g}-b \geq n \in \mathbb{N}$,

$$
\begin{aligned}
f_{B}^{(n, b)}(g) & =(q+1) q^{n-1-(a-b) / 2} \sum_{\substack{f\left(\mu^{-1} \nu\right)=n \leq k_{B, g}-b \\
\mu^{a} \nu^{b}=1}} \mu\left(o_{1}^{-1}\right) \nu\left(o_{2}^{-1}\right) \\
& =(q+1) q^{n-1-(a-b) / 2}\left(\tilde{f}_{a b}^{(n)}(g)-\tilde{f}_{a b}^{(n-1)}(g)\right),
\end{aligned}
$$

where for $n \in \mathbb{N} \cup\{0\}$,

$$
\tilde{f}_{a b}^{(n)}(g)=\sum_{\substack{0 \leq f\left(\mu^{-1} \nu\right) \leq n \leq k_{B, g}-b \\ \mu^{a} \nu^{b}=1}} \mu\left(o_{1}^{-1}\right) \nu\left(o_{2}^{-1}\right) .
$$


For $n \in \mathbb{N} \cup\{0\}$, let $X_{a b}^{(n)}=\left\{(\mu, \nu) \mid \mu, \nu \in \operatorname{Hom}_{\text {cts }}\left(\mathfrak{o}^{\times}, \mathbb{C}^{\times}\right), 0 \leq f\left(\mu^{-1} \nu\right) \leq\right.$ $\left.n, \mu^{a} \nu^{b}=1\right\}$. Then clearly $\tilde{f}_{a b}^{(n)}(g)=0$ if $n>k_{B, g}-b$, and if $n \leq k_{B, g}-b$ we have

$$
\tilde{f}_{a b}^{(n)}(a, b)= \begin{cases}0, & \text { if } \exists(\mu, \nu) \in X_{a b}^{(n)} \text { with } \mu\left(o_{1}^{-1}\right) \nu\left(o_{2}^{-1}\right) \neq 1, \\ \# X_{a b}^{(n)}, & \text { otherwise. }\end{cases}
$$

Using that $\mathfrak{o}^{\times} / 1+\mathfrak{p}^{n}$ is, as an abstract group, the direct product of a cyclic group of order $(q-1)$ and a group of order $q^{n-1}$, and also that for $n \geq 1, f\left(\mu^{-1} \nu\right) \leq$ $n \Longleftrightarrow f(\mu) \leq n$ and $f(\nu) \leq n$ we have

$$
\# X_{a b}^{(n)}= \begin{cases}(q-1, r), & \text { if } n=0, \text { and } \\ (q-1)(a, b, q-1) \cdot q^{n-1}, & \text { if } n \geq 1 .\end{cases}
$$

Further, we define $l_{a b}(g)$ to be the largest $n \in \mathbb{N} \cup\{0\}$, if it exists, such that $\mu\left(o_{1}\right) \nu\left(o_{2}\right)=1$ for all $\mu, \nu \in X_{a b}^{(n)}$. If no such $n$ exists we define $l_{a b}(g)=-1$. As we shall shortly see, $l_{a b}(g)$ is going to figure into our formulas, so let us describe $l_{a b}(g)$ explicitly in terms of $o_{1}$ and $o_{2}$. Fix a surjection $\mathfrak{o}^{\times} \rightarrow \mathbb{F}_{q}^{\times}$with kernel $1+\mathfrak{p}$, and for any $o \in \mathfrak{o}^{\times}$let $\dot{o}$ denote its image under the surjection. If $\dot{o}_{1} \dot{o}_{2}$ is not a power of $r$, then $l_{a b}(g)=-1$. Else if $\left(\dot{o}_{1}, \dot{o}_{2}\right)$ is not of the form $\left(\dot{o}^{a}, \dot{o}^{b}\right)$ for any $\dot{o} \in \mathbb{F}_{q}^{\times}$, then $l_{a b}(g)=0:$ since $\mathfrak{o}^{\times} / 1+\mathfrak{p} \cong \mathbb{F}_{q}^{\times}$is cyclic, this is precisely the condition to ensure $\mu\left(o_{1}\right) \nu\left(o_{2}\right) \neq 1$ for at least one $(\mu, \nu) \in X_{a b}^{(1)}$. Else,

$$
l_{a b}(g)=\operatorname{val}\left(\left(\frac{\operatorname{det} \operatorname{Sym}^{a+b} g}{(\operatorname{det} g)^{b}}\right)^{q-1}-1\right) .
$$

This easily follows from the fact that for any $n \in \mathbb{N}, \mathfrak{o}^{\times} / 1+\mathfrak{p}^{n}$ is a direct product of a cyclic group of order $q-1$ and a $p$-group.

Thus, using equations (5.8), (5.9) and (5.10), we get that $f_{B}^{(n, b)}(g)$, for $g$ conjugate to $\left(\varpi^{a} o_{1}, \varpi^{b} o_{2}\right)$ and $n \geq 1$, equals:

(i) 0 , if $n>\min \left(l_{a b}(g)+1, k_{B, g}-b\right)$;

(ii) $(q+1) q^{-(a-b) / 2}((q-1)(a, b, q-1)-(q-1, r))$, if $1=n \leq \min \left(k_{B, g}-\right.$ $\left.b, l_{a b}(g)\right)$

(iii) $-(q+1) q^{-(a-b) / 2}(q-1, r)$, if $1=n=l_{a b}(g)+1 \leq k_{B, g}-b$;

(iv) $(q-1)\left(q^{2}-1\right)(a, b, q-1) q^{2 n-3-(a-b) / 2}$, if $2 \leq n \leq \min \left(k_{B, g}-b, l_{a b}(g)\right)$; and

(v) $-\left(q^{2}-1\right)(a, b, q-1) q^{2 n-3-(a-b) / 2}$, if $2 \leq n=l_{a b}(g)+1 \leq k_{B, g}-b$.

Therefore by equation (5.7) (recall we have fixed our $a$ and $b$ and $g): f_{B}^{(>0)}(g)=$ $f_{B}^{(>0, b)}(g)$ can be given by:

(i) If $k_{B, g}-b \leq 0$ or $l_{a b}(g)=-1$, then $f_{B}^{(>0)}(g)=0$.

(ii) If $l_{a b}(g)=0$ and $k_{B, g}-b>0$, then $f_{B}^{(>0)}(g)=-(q+1) q^{-(a-b) / 2}(q-1, r)$.

(iii) If $0<k_{B, g}-b \leq l_{a b}(g)$, then

$$
\begin{aligned}
f_{B}^{(>0)}(g)= & (q+1) q^{-(a-b) / 2}((q-1)(a, b, q-1)-(q-1, r)) \\
& \quad+\sum_{n=2}^{k_{B, g}-b}(q-1)(a, b, q-1)\left(q^{2 n-1-(a-b) / 2}-q^{2 n-3-(a-b) / 2}\right) \\
= & q^{-(a-b) / 2}\left(-(q+1)(q-1, r)+(q-1)(a, b, q-1)\left(1+q^{2 k_{B, g}-2 b-1}\right)\right) .
\end{aligned}
$$


(iv) If $0<l_{a b}(g)<k_{B, g}-b$, then

$$
\begin{aligned}
f_{B}^{(>0)}(g)=(q+1) q^{-(a-b) / 2}((q-1)(a, b, q-1)-(q-1, r)) \\
\quad+\sum_{n=2}^{l_{a b}(g)}(q-1)(a, b, q-1)\left(q^{2 n-1-(a-b) / 2}-q^{2 n-3-(a-b) / 2}\right) \\
\quad-\left(q^{2}-1\right)(a, b, q-1) q^{2 l_{a b}(g)-1-(a-b) / 2} \\
=q^{-(a-b) / 2}\left(-(q+1)(q-1, r)+(q-1)(a, b, q-1)\left(1-q^{2 l_{a b}(g)}\right)\right) .
\end{aligned}
$$

Note that the formulas for (ii) and (iv) are so compatible as to admit filing them under the one case of $0 \leq l_{a b}(g)<k_{B, g}$.

\section{Putting things together}

Now we are in a position to compute the the function $f_{B} \in C_{c}^{\infty}(G)$ that realizes $z_{r} * \operatorname{ch}_{K_{B}}$, the convolution product of our distribution $z_{r}$ with the measurenormalized characteristic function of an open compact subgroup of $G$ of the form $1+B$, where $B$ is a lattice in $\mathbf{M}_{2}(F)$ satisfying Hypothesis 2.1.

Theorem 6.1. Let $g \in G$. Set $b=\operatorname{val}(\operatorname{tr} g)$ and $a=r-b$. Then $f_{B}(g)=0$ if $\operatorname{val}(\operatorname{det} g) \neq r$. In the case $\operatorname{val}(\operatorname{det} g)=r$ we have the following results:

(i) If $b<0$ or $k_{B, g} \leq 0$, then $f_{B}(g)=0$.

(ii) If $\min (b,\lceil r / 2\rceil) \geq k_{B, g} \geq 0$, then

$$
f_{B}(g)=-\chi_{r}(g)(q-1, r) \frac{q^{2 k_{B, g}}-1}{q^{r / 2}} .
$$

(iii) If $\min \left(b, k_{B, g}\right) \geq\lceil r / 2\rceil$, then

$$
f_{B}(g)=-\chi_{r}(g)(q-1, r) \frac{q^{r+1}-1}{q^{r / 2}} .
$$

(iv) If $0 \leq b<\min \left(k_{B, g},\lceil r / 2\rceil\right)$ and $l_{a b}(g)=0$, then

$$
f_{B}(g)=\frac{-(q+1)(q-1, r)+\chi_{r}(g)(q-1, r)\left(q^{b+1}+q^{-b}\right)}{q^{(r / 2)-b}} .
$$

(v) If $0 \leq b<\min \left(k_{B, g},\lceil r / 2\rceil\right)$ and $l_{a b}(g) \geq k_{B, g}-b$, then $f_{B}(g)$ equals $\frac{-(q+1)(q-1, r)+(q-1)(a, b, q-1)\left(q^{2 k_{B, g}-2 b-1}+1\right)+(q-1, r)\left(q^{b+1}+q^{-b}\right)}{q^{(r / 2)-b}}$.

(vi) If $0 \leq b<\min \left(k_{B, g},\lceil r / 2\rceil\right)$ and $0 \leq l_{a b}(g)<k_{B, g}-b$, then $f_{B}(g)$ equals $\frac{-(q+1)(q-1, r)+(q-1)(a, b, q-1)\left(1-q^{2 l_{a b}(g)}\right)+(q-1, r)\left(q^{b+1}+q^{-b}\right)}{q^{(r / 2)-b}}$.

(vii) If $0 \leq b \leq \min \left(k_{B, g},\left\lceil\frac{r}{2}\right\rceil\right)$ and $l_{a b}(g)=-1$, then

$$
f_{B}(g)=\frac{\chi_{r}(g)(q-1, r)\left(q^{b+1}+q^{-b}\right)}{q^{(r / 2)-b}}=0 .
$$

Proof. This follows, by using $f_{B}=f_{B}^{(0)}+f_{B}^{(>0)}$, the value of $f_{B}^{(0)}$ from equation (4.9) and the computation of $f_{B}^{(>0)}$ above. In (v) and (vi) we see that if $l_{a b}(g)>0$, then automatically $\chi_{r}(g)=1$, something readily verified. The vanishing in (vii) follows from the explicit description of $l_{a b}(g)$ given before. 
Remark 6.2. Scholze in 9 ] had considered the case $r=1$, and normalized the Haar measure on $G$ giving $\mathbf{G L}_{2}(\mathfrak{o})$ volume $q-1$ instead of 1 . Furthermore, the exact distribution he considered was $q^{1 / 2}$ times the one we considered. Keeping this in mind, it is straightforward to check that upon writing $r=1$ our Theorem 6.1 agrees with his computations.

\section{ACKNOWLEDGEMEnTS}

I thank Professor Robert Kottwitz for suggesting this problem, for sharing some of his insights and perspectives and for his patient guidance and encouragement. I also thank the referee for making suggestions that helped improve the precision and articulation of many-a-point in this paper, and for pointing out several misprints.

\section{REFERENCES}

[1] Anne-Marie Aubert and Roger Plymen, Explicit Plancherel formula for the p-adic group GL $(n)$, C. R. Math. Acad. Sci. Paris 338 (2004), no. 11, 843-848, DOI 10.1016/j.crma.2004.03.026 (English, with English and French summaries). MR2059659 (2005a:22010)

[2] J. N. Bernstein, Le "centre" de Bernstein, Representations of reductive groups over a local field, Travaux en Cours, Hermann, Paris, 1984, pp. 1-32 (French). Edited by P. Deligne. MR771671 (86e:22028)

[3] T. Haines and B. C. Ngô, Nearby cycles for local models of some Shimura varieties, Compositio Math. 133 (2002), no. 2, 117-150, DOI 10.1023/A:1019666710051. MR.1923579 (2003h:11065)

[4] Thomas J. Haines, Introduction to Shimura varieties with bad reduction of parahoric type, Harmonic analysis, the trace formula, and Shimura varieties, Clay Math. Proc., vol. 4, Amer. Math. Soc., Providence, RI, 2005, pp. 583-642. MR2192017(2006m:11085)

[5] Robert E. Kottwitz, Points on some Shimura varieties over finite fields, J. Amer. Math. Soc. 5 (1992), no. 2, 373-444, DOI 10.2307/2152772. MR1124982 (93a:11053)

[6] Allen Moy and Marko Tadić, The Bernstein center in terms of invariant locally integrable functions, Represent. Theory 6 (2002), 313-329 (electronic), DOI 10.1090/S1088-4165-0200181-4. MR1979109 (2004f:22019)

[7] Allen Moy and Marko Tadić, Erratum to: The Bernstein center in terms of invariant locally integrable functions, Represent. Theory 9 (2005), 455-456 (electronic). MR2167901

[8] Michael Rapoport, A guide to the reduction modulo $p$ of Shimura varieties, Astérisque 298 (2005), 271-318 (English, with English and French summaries). Automorphic forms. I. MR2141705 (2006c:11071)

[9] Peter Scholze, The Langlands-Kottwitz approach for the modular curve, Int. Math. Res. Not. IMRN 15 (2011), 3368-3425, DOI 10.1093/imrn/rnq225. MR2822177(2012k:11090)

[10] G. van Dijk, Computation of certain induced characters of $\mathfrak{p}$-adic groups, Math. Ann. 199 (1972), 229-240. MR0338277 (49 \#3043)

[11] J.-L. Waldspurger, La formule de Plancherel pour les groupes p-adiques (d'après HarishChandra), J. Inst. Math. Jussieu 2 (2003), no. 2, 235-333, DOI 10.1017/S1474748003000082 (French, with French summary). MR 1989693(2004d:22009)

Department of Mathematics, University of Chicago, 5734 University Avenue, Chicago, ILLINOIS 60637

Current address: School of Mathematics, Tata Institute of Fundamental Research, Colaba, Mumbai - 400 005, India

E-mail address: sandeepv@math.tifr.res.in 